\title{
Intracellular Cholesterol Trafficking and Impact in Neurodegeneration
}

\author{
Fabian Arenas $^{1,2,3}$, Carmen Garcia-Ruiz ${ }^{1,2,3,4}$ and Jose C. Fernandez-Checa 1, 2, 3, 4* \\ 'Department of Cell Death and Proliferation, Instituto de Investigaciones Biomédicas de Barcelona, Consejo Superior de \\ Investigaciones Científicas, Barcelona, Spain, ${ }^{2}$ Liver Unit and Hospital Clinic I Provincial, IDIBAPS, Barcelona, Spain, ${ }^{3}$ Centro \\ de Investigación Biomédica en Red, CIBEREHD, Barcelona, Spain, ${ }^{4}$ Southern California Research Center for ALDP and \\ Cirrhosis, Los Angeles, CA, United States
}

\section{OPEN ACCESS}

Edited by:

Andrei Surguchov,

Kansas University of Medical Center Research Institute, United States

Reviewed by:

Anthony John Hannan,

Florey Institute of Neuroscience and

Mental Health, Australia

Christian Gonzalez-Billault,

Universidad de Chile, Chile

${ }^{*}$ Correspondence:

Jose C. Fernandez-Checa checa229@yahoo.com

Received: 25 July 2017 Accepted: 02 November 2017

Published: 17 November 2017

Citation:

Arenas F, Garcia-Ruiz C and

Fernandez-Checa JC (2017)

Intracellular Cholesterol Trafficking and Impact in Neurodegeneration.

Front. Mol. Neurosci. 10:382.

doi: 10.3389/fnmol.2017.00382
Cholesterol is a critical component of membrane bilayers where it plays key structural and functional roles by regulating the activity of diverse signaling platforms and pathways. Particularly enriched in brain, cholesterol homeostasis in this organ is singular with respect to other tissues and exhibits a heterogeneous regulation in distinct brain cell populations. Due to the key role of cholesterol in brain physiology and function, alterations in cholesterol homeostasis and levels have been linked to brain diseases and neurodegeneration. In the case of Alzheimer disease (AD), however, this association remains unclear with evidence indicating that either increased or decreased total brain cholesterol levels contribute to this major neurodegenerative disease. Here, rather than analyzing the role of total cholesterol levels in neurodegeneration, we focus on the contribution of intracellular cholesterol pools, particularly in endolysosomes and mitochondria through its trafficking via specialized membrane domains delineated by the contacts between endoplasmic reticulum and mitochondria, in the onset of prevalent neurodegenerative diseases such as AD, Parkinson disease, and Huntington disease as well as in lysosomal disorders like Niemann-Pick type C disease. We dissect molecular events associated with intracellular cholesterol accumulation, especially in mitochondria, an event that results in impaired mitochondrial antioxidant defense and function. A better understanding of the mechanisms involved in the distribution of cholesterol in intracellular compartments may shed light on the role of cholesterol homeostasis disruption in neurodegeneration and may pave the way for specific intervention opportunities.

Keywords: cholesterol, lysosomes, mitochondria, endoplasmic reticulum, mitochondria associated ER membranes, Alzheimer disease, Parkinson disease, Niemann Pick type C disease

\section{INTRODUCTION}

As amphipathic sterol, cholesterol exerts key structural and physiological functions in all cells. Its planar and rigid structure regulates the fluidity and permeability of the phospholipid bilayer and for the solutes and ions, respectively (Segatto et al., 2014). Moreover, the functions of membrane proteins are modulated by cholesterol, which also participates in many membrane trafficking and transmembrane signaling processes (Egawa et al., 2015). Accordingly, the plasma membrane (PM) of all eukaryotic cells have the cholesterol as both structural and functional essential component, since also works as a precursor for the biosynthesis of bile acids, steroid hormones, and vitamin D which, in turn, have important biological roles as signal transducers and lipid solubilizers (van der Wulp et al., 2013). 
Outside the central nervous system (CNS), cellular cholesterol requirement is assured by its de novo synthesis and intracellular uptake from lipoprotein-containing particles derived from the diet. However, because the blood-brain barrier (BBB) is impermeable to the plasma lipoproteins, nearly all cholesterol existing in the brain is produced by de novo synthesis (Zhang and Liu, 2015). In addition, the degradation and excretion of cholesterol from the brain is mainly driven by the neuronspecific cytochrome P450 oxidase CYP46A1, which hydroxylates cholesterol to 24S-hydroxycholesterol (24-OHC), the most abundant oxysterol in the brain (Gamba et al., 2015), that unlike cholesterol, can cross the $\mathrm{BBB}$, entering the circulation for its disposal by the liver.

Although, the brain makes up only $2.1 \%$ of body weight, it contains $23 \%$ of the total body cholesterol and exhibits a 10 fold higher cholesterol concentration compared to other tissues (Vance, 2012). Most (at least 99\%) of the brain cholesterol is present in an unesterified form, which is distributed in two major pools: (i) Close to $70 \%$ is present in the myelin sheaths of oligodendrocytes (white matter); and (ii) and the $30 \%$ remainder is present within the plasmalemmal and subcellular membranes of astrocytes and neurons (gray matter). Across the different cell types of the CNS de novo synthesis of cholesterol is not homogenous. For instance, oligodendrocytes (responsible for axon myelinization) have a higher capacity for cholesterol biosynthesis than astrocytes, which in turn, exhibit at least 2- to 3-fold higher capacity than neurons (van der Wulp et al., 2013; Segatto et al., 2014; Egawa et al., 2015). During the perinatal period and adolescence the neurons needs encircled in myelin and consequently is when most of brain cholesterol accumulates. After myelination, the whole bulk of cholesterol in the adult brain is maintained at a very low levels with minimal loss (half-life up to 5 years), although it has been suggested that the turnover rate could vary between the different cell types of the CNS, being very high in some neurons with turnover estimates of about $20 \%$ per

Abbreviations: $\mathrm{ABC}$, ATP-binding cassette transporters; $\mathrm{ABCA}, \mathrm{ABC}$ subfamily A transporters; $A \beta$, amyloid- $\beta$ peptide; ACAT1, acylcoenzyme A:cholesterol acyltransferase 1; AD, Alzheimer disease; ANT, adenine nucleotide transporter; ApoA-I, apolipoprotein A-I; ApoE, apolipoprotein E; ApoER2, ApoE receptor 2; APP, amyloid precursor protein; APP/PS1/SREBP2, APP/PS1 mice overexpressing SREBP2; ATAD3, AAA domain-containing protein 3; BACE1, Beta-secretase 1; BBB, blood brain barrier; CAV, caveolins; CatD, cathepsin D; CNS, central nervous system; CSF, cerebrospinal fluid; ER, endoplasmic reticulum; HD, Huntington disease; HDL, high-density lipoproteins; HMG-CoA, 3hydroxy-3-methylglutaryl-CoA; HMGR, HMG-CoA reductase; IMM, inner mitochondrial membrane; LDLR, low-density lipoprotein receptor; LE/LY, late endosome/lysosome; LRP-1, lipoprotein receptor-related protein 1; LRO, lysosome-related organelle; LXR, nuclear liver X receptor; MAM, mitochondria associated ER membranes; MCS, membrane contact sites; MS, multiple sclerosis; mtDNA, mitochondrial DNA; mGSH, mitochondrial GSH; NPC1, NiemannPick type $\mathrm{C} 1$ protein; NPC2, Niemann-Pick type C2 protein; OGC, 2oxoglutarate carrier; OSBP, oxysterol-binding protein; ORPs, OSBP-related proteins; OMM, outer mitochondrial membrane; PD, Parkinson disease; PM, plasma membrane; PI4P, phosphatidylinositol 4-phosphate; PSEN1/2, presenilins $1 / 2$; ROS, reactive oxygen species; SCAP, SREBP cleavage activating protein; SREBP2, sterol regulatory element protein 2; StAR, steroidogenic acute regulatory protein; START, StAR-related domain lipid transfer; TSPO, Translocator protein; VDAC, voltage-dependent anion channel; VLDL, very low-density lipoproteins; $\mathrm{MPP}+$, 4-methylpyridinium; 24-OHC, 24-hydroxycholesterol; 24-DHCR, 24dehydrocholesterol reductase; 7-DHCR, 7-dehydrocholesterol reductase. day (Moutinho et al., 2016). Besides the cell-specific rate of brain cholesterol synthesis and turnover, there is evidence indicating a region-dependence of cholesterol synthesis, which is influenced by aging and gender (Segatto et al., 2014). Thus, alterations in the exquisite feedback mechanisms that regulate brain cholesterol homeostasis could be either the cause or the consequence of a number of neurodegenerative disorders.

As the contribution of the absolute levels of cholesterol in major prevalent neurodegenerative diseases, such as Alzheimer disease $(\mathrm{AD})$, is controversial, as discussed below, in this review we focus on the putative contribution of intracellular cholesterol pools, particularly in the endolysosomes and mitochondria, in the development of neurodegeneration. A further understanding of the mechanisms and functional consequences associated with the disruption in the intracellular cholesterol trafficking and accumulation may provide novel opportunities for the treatment of major neurological diseases.

\section{CHOLESTEROL METABOLISM IN THE CNS}

\section{Biosynthesis, Homeostasis, and Turnover}

Although, all mammalian cells have the capacity to biosynthesize cholesterol de novo, this process is crucial for the CNS since cholesterol's metabolism in the brain occurs isolated to the rest of the organism due to the $\mathrm{BBB}$. Cellular cholesterol is synthesized primarily in the endoplasmic reticulum (ER) from acetate in a complex and resource-intense process that involves over 30 enzymatic steps, initiated by the conversion of acetylCoA to 3-hydroxy-3-methylglutaryl-CoA (HMG-CoA) and then irreversibly converted to mevalonate by the rate-limiting enzyme, HMG-CoA reductase (HMGR). This is followed by multi-step enzymatic reactions that sequentially convert mevalonate into 3-isopenenyl pyrophosphate, farnesyl pyrophosphate, squalene, lanosterol, and finally to cholesterol in a 19-step process, involving two distinct but related pathways (Figure 1). The Bloch and Kandutsch-Russel pathways use desmosterol and 7-hydroxycholesterol as the immediate precursors of cholesterol through their reduction by 24 -dehydrocholesterol reductase (DHCR24) and 7-dehydrocholesterol reductase (DHCR7), respectively (Martin et al., 2014). Mutations in the genes encoding DHCR24 and DHCR7 cause rare hereditary neurological diseases, illustrating the importance of cholesterol homeostasis in brain physiology (Figure 1, see below). Unlike axons, de novo synthesis of cholesterol occurs in neuronal somata of adult neurons, which have minor capability than astrocytes to produce cholesterol. Thus, in neurons the newly synthesized cholesterol needed an anterograde soma-to-axon transport (Zhang and Liu, 2015).

The cholesterol biosynthesis has a crucial feedback control that involves sterol-mediated degradation of HMGR (Figure 1). Accumulation of sterols in the ER membranes triggers the ubiquitination of HMGR by Insig-1 and Insig- 2 and the subsequent proteasome-mediated degradation of HMGR. In addition, HMGR gene expression is regulated by ER-bound membrane transcription factor sterol regulatory element protein 2 (SREBP2), whose activation depends on the sterol-sensitive SREBP cleavage activating protein (SCAP). The ER-sterol 


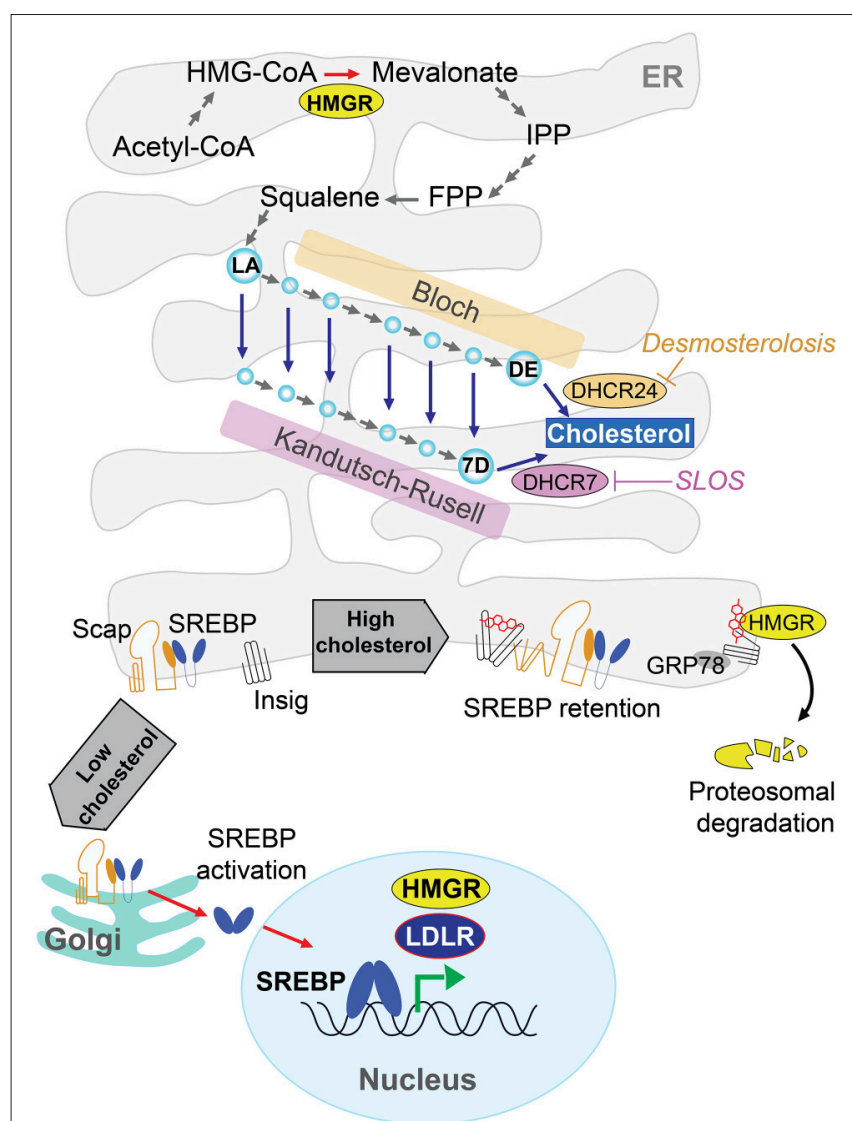

FIGURE 1 | De novo cholesterol synthesis in the brain. Cells synthesize cholesterol at endoplasmic reticulum (ER) from acetyl-CoA through several energy demanding enzymatic steps. The rate-limiting enzyme 3-hydroxy-3-methylglutaryl-CoA (HMG-CoA) reductase (HMGR) converts irreversibly HMG-CoA to mevalonate, followed by multi-step enzymatic reactions until lanosterol $(L A)$ and finally to cholesterol by 19-step process involving the Bloch and Kandutsch-Russel pathways. These pathways use desmosterol (DE) or 7-dehydroxycholesterol (7D) as precursors of cholesterol through DHCR24 and DHCR7, respectively. Mutations in these genes cause rare hereditary neurological disease such as Desmosterolosis or Smith-Lemli-Opitz syndrome (SLOS). The cholesterol biosynthesis has a sterol-dependent feedback control that modulates the HMGR levels. ER-sterol accumulation triggers both proteasome-mediated degradation of HMGR through an Insig/GRP78-dependent mechanism and the ER-retention of sterol regulatory element protein 2 (SREBP2), which in turn is controlled by sterol-sensitive SREBP cleavage activating protein (SCAP).

containing is sensed by SCAP to retain full-length SREBP2 at the ER. Upon ER-sterol depletion, SCAP interacts with SREBP2 to exit the ER and move to the Golgi apparatus, where SREBP2 is sequentially cleaved by the specific proteases S1P and S2P. This event releases the soluble $\mathrm{N}$ terminus domain of SREBP2, which travels to the nucleus to induce the transcription of HMGR as well as low-density lipoprotein receptor (LDLR; Garcia-Ruiz et al., 2009; Martin et al., 2014; Fernández-Checa, 2015).

The cellular machinery that responds to an excess of cholesterol is expressed at higher levels in neurons than astrocytes. Thus, neurons may handle cholesterol overload by different mechanisms, involving cholesterol esterification and the concomitant intracellular storage in lipid droplets, direct excretion through ATP-binding cassette (ABC) transporters or conversion to 24-OHC through CYP46A1 (Figure 2). The esterification of cholesterol is catalyzed by acylcoenzyme A:cholesterol acyltransferase 1 (ACAT1/SOAT1) in the ER (Zhang and Liu, 2015) and the accumulation of cholesterol esters in lipid droplets represents up to $1 \%$ of the total cholesterol content in neurons. However, in conditions where cholesterol homeostasis is disrupted due for instance to the lack of ApoE or cholesterol overload, ACAT1/SOAT1 becomes active in astrocytes (Karten et al., 2006). In addition, it has been observed in Drosophila that the accumulation of lipid droplets in glia is a consequence of reactive oxygen species (ROS) and mitochondrial defects (Liu et al., 2015). These findings imply that glial lipid droplets accumulation related with mitochondrial defects in neurons could be an evolutionarily conserved process, emerging as a biomarker for neurodegenerative disease at early stages (Liu et al., 2015).

Neurons express proteins of the ABC subfamily A transporters (ABCA) to control the cellular cholesterol efflux at PM's level (Figure 2). This pathway for cholesterol elimination from neurons is independent of CYP46A1. Compared to astrocytes, the higher expression of ABCA1 in neurons mediates the cholesterol efflux to both lipid-free apolipoprotein A-I (ApoAI) and $\mathrm{E}$ (ApoE) present in cerebrospinal fluid (CSF). These lipoproteins, in turn, can be removed from the brain through lipoprotein receptor-related protein 1 (LRP1; Koldamova et al., 2014). Recently, it has been described that LRP1 is crucial to limit intracellular cholesterol accumulation through inhibition of cholesterol biosynthesis via TGF $\beta$-induced enhanced Wnt5a expression, increasing cholesterol export by stimulation of ABCA1 expression (El Asmar et al., 2016). Interestingly, the Wnt signaling pathway prevents mitochondrial dysfunction in neurons and protects from neurodegeneration (Arrazola et al., 2015).

On the other hand, the hydroxylation of cholesterol to 24OHC by CYP46A1 constitutes the major excretion pathway of cholesterol in the brain (40-50\% of brain cholesterol turnover) since unlike non-oxidized cholesterol, 24-OHC can cross BBB at very higher rate than cholesterol itself (Figure 2). Interestingly, the induction of the activation of nuclear liver $\mathrm{X}$ receptor (LXR) by 24-OHC can upregulate ABCA1's expression, which can influence whole-brain cholesterol homeostasis (Zhang and Liu, 2015). Restricted to somata and dendrites (not in axons or presynaptic terminals) of pyramidal cells of the cortex and Purkinje cells of cerebellum (almost undetectable in astrocytes), the expression of CYP46A1 is located mainly in the ER (Moutinho et al., 2016). Additionally, both the plasma and CSF levels of 24-OHC are altered in several neurodegenerative disorders, such as AD, multiple sclerosis (MS), Parkinson disease (PD), and Huntington disease (HD; Moutinho et al., 2016).

\section{Cholesterol Transport}

The requirement of neurons for cholesterol is very high, as it is used in neurite formation, maintenance, and synaptic connectivity. Adult neurons meet their cholesterol needs from the generation of cholesterol from astrocytes, which are able to 
secrete cholesterol-rich lipoproteins containing ApoE (the main lipid transporter in the CNS) that are taken by neurons via endocytosis mediated by LDLR and LRP1 (Figure 2). Notably, the expression of LDLR family is particularly enriched in neurons in specific brain subcellular sites. For instance, multiple cell types of the BBB (e.g., pericytes, neurons, and astrocytes) express LRP1 principally at the abluminal side (Zlokovic, 2010), where it is able to recognize and participate in the endocytosis of several ligands including ApoE, amyloid precursor protein (APP), and amyloid- $\beta$ peptide (A $\beta$; Ramanathan et al., 2015).

On the other hand, chronic high levels of total serum cholesterol increase the brain expression and secretion of ApoE from glial cells (Petanceska et al., 2003). Among the three major human isoforms of ApoE, ApoE3 is most representative isoform (78\%), while ApoE4 (15\%) and ApoE2 (8\%) isoforms are less representatives in humans (El Gaamouch et al., 2016). The affinity to ApoE-receptors and lipoprotein subtypes vary functionally between human ApoE isoforms, which differ in two residues: small, phospholipid-rich HDLs are recognized by ApoE2 and ApoE3; while ApoE4 binds to large, triglyceriderich VLDL. On the other hand, glia, neurons, and brain endothelial cells (BECs) express LDLR and LRP-1 as endocytic receptors, while VLDL receptor (VLDLR) and LRP8/ApoE receptor 2 (ApoER2) function as signaling receptors. In addition, lipidation grade of ApoE can also modify the specificity of its ApoE-receptor binding (El Gaamouch et al., 2016). Thus, in addition to regulating cholesterol/lipid homeostasis in the CNS, ApoE modulates multiple mechanistic pathways that

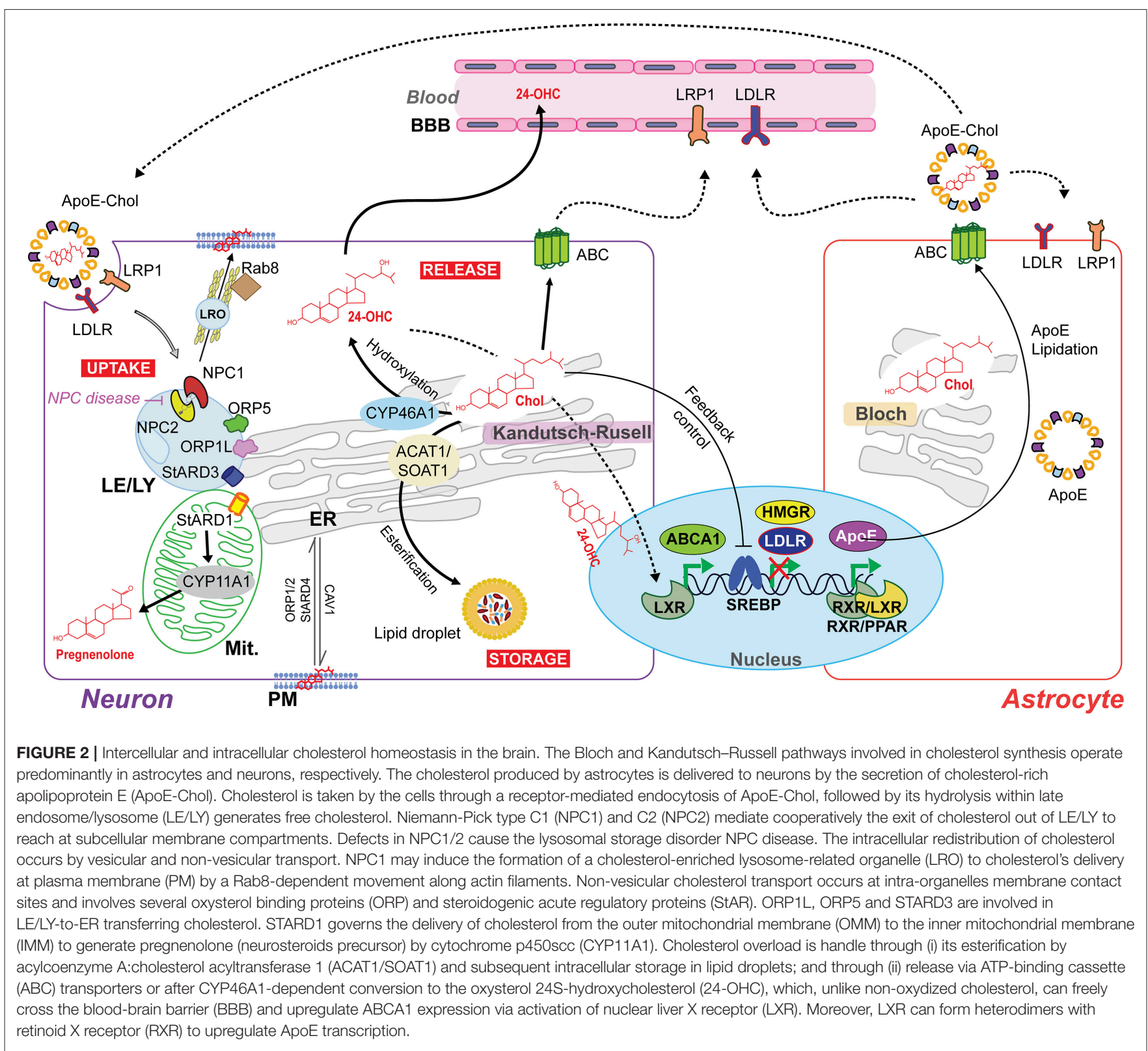


collectively affect neurogenesis, glucose metabolism, synaptic function, cognition, mitochondrial function, neuronal atrophy, neuroinflammation, tau phosphorylation, and metabolism and aggregation of $\mathrm{A} \beta$, which are of relevance for $\mathrm{AD}$.

\section{Intracellular Cholesterol Distribution and Trafficking between Organelles}

Once synthesized in the ER, cholesterol is then distributed to different membrane bilayers in the cell. This redistribution in different subcellular compartments occurs by an arrangement between vesicle-mediated inter-organelle cholesterol transport and protein-mediated (i.e., non-vesicular) monomeric cholesterol transfer through the aqueous cytoplasm (Zhang and Liu, 2015). Vesicular trafficking involves the incorporation of cholesterol into the membranes of transport vesicles that migrates along the cytoskeletal tracks when metabolic energy is available. Non-vesicular transport, however, requires sterol transfer proteins, which pack cholesterol in a hydrophobic pocket that shuttles through the aqueous cytosol between membranes, especially those that are anchored together by specialized proteins (Mesmin et al., 2013).

A number of proteins, including members of steroidogenic acute regulatory protein (StAR)-related domain lipid transfer (START) family, and oxysterol-binding protein (OSBP)-related proteins (ORPs), are thought to contribute to the non-vesicular transport of cholesterol within the aqueous environment of the cytosol, and between organelles and the PM (Figure 2; Lev, 2010; Mesmin et al., 2013). Human START proteins are a family of 15 members, sharing a conserved C-terminal with a 210-residue hydrophobic binding pocket able to accommodate a single lipid molecule that ingress and egress by an $\alpha$-helix that operates as a "lid" (Thorsell et al., 2011). Among them, the members that bind sterols are STARD1, STARD3, and STARD4 subfamily (Soffientini and Graham, 2016; Elustondo et al., 2017). The second group of lipid-binding/sensing proteins includes the ORPs that have a conserved C-terminal OSBP-homology domain, and bind cholesterol, ergosterol, oxysterols, phosphatidylinositol-4phosphate, and/or phosphatidylserine (Olkkonen and Li, 2013). Sterol-binding, sterol-sensing and trafficking proteins influence the expression and activity of sterol-responsive transcription factors, regulating the expression of genes-encoding proteins that synthesize, metabolize and release cholesterol, and direct the trafficking of cholesterol between the PM, mitochondria, Golgi and endosomal/lysosomal compartments, determining the fate of the cholesterol (El Gaamouch et al., 2016; Soffientini and Graham, 2016).

Before it reaches subcellular membrane compartments, externally endocytosed ApoE-cholesterol complex enters late endosome/lysosome (LE/LY) where the ApoE-containing cholesterol ester is hydrolyzed by acid lipase to generate free cholesterol, which is then distributed primarily to the PM as well as to the ER via Niemann-Pick type C1 (NPC1) and C2 (NPC2) protein-dependent mechanisms (Figure 2; FernándezCheca, 2015). NPC2 first binds the hydrophobic side chain of unesterified cholesterol and hands over the molecule to the
N-terminal domain of NPC1, which inserts cholesterol into the lysosomal membrane. Although, the precise mechanism by which NPC1 mediates cholesterol egress from LE to the ER and $\mathrm{PM}$ is not fully understood, recently it has been proposed that NPC1 seems to promote a cholesterol-enriched lysosome-related organelle (LRO) formation able to move along actin filaments in a Rab8- and myosin-5-dependent manner toward the PM (Luo et al., 2017). Furthermore, although oxysterol binding proteins such as OPR5 work directly with NPC1, recent findings uncovered an interaction between RID $\alpha$ and ORP5 that could bypass NPC1 function in the presence of NPC2, which represents a minor route for cholesterol egress from lysosomes (Cianciola et al., 2013). An excess of PM's cholesterol triggers its retrograde transport to the ER by a STARD4, ORP1, and ORP2-dependent process (Luo et al., 2017). In addition, the transfer of cholesterol from LE/LY-to-ER seems to occur by key components of some ER-LE membrane contact sites (MCS) bridging complexes, which include cholesterol-binding proteins such as ORP1L, ORP5, and STARD3 (Luo et al., 2017). Thus, an excess of cholesterol content in ER triggers a feedback mechanism that regulate cholesterol homeostasis at the transcriptional and post-transcriptional levels, including blockage of SREBP/SCAP ER-to-Golgi transport, proteasomal degradation of HMGCR, and activation of ACAT1 to produce cholesteryl esters stored in lipid droplets (Figure 2). In addition, ORPs act as transporters and/or lipid sensors at MCS, exemplified by the exchange of cholesterol and PI4P at the ER-Golgi contact site (Olkkonen and Li, 2013; Goto et al., 2016).

\section{Membrane Lipid Rafts}

Once distributed in organelles, intracellular cholesterol not only generates a semipermeable barrier between cellular compartments that regulates membrane fluidity, it also induces the formation of cholesterol-enriched microdomains of the membrane called membrane "lipid-rafts." Besides cholesterol, lipid-rafts are characterized by the presence of sphingolipids and specific scaffolding proteins that act as platforms for signal transduction, cytoskeletal organization, and vesicular trafficking (Egawa et al., 2015). Of particular relevance for lipid-rafts are the presence of caveolins (CAV), essential components of caveolae, which exhibit both scaffolding- and cholesterolbinding functions. Non-caveolar pools of CAVs are presents in myriad locations, including endosomes, ER, lipid droplets, and mitochondria (Bosch et al., 2011). CAVs regulate many receptors, including neurotransmitter receptors (NMDA and AMPA receptors), signaling proteins that trigger the production of cAMP (G protein-coupled receptors, adenylyl cyclases, and phosphodiesterases), and receptor tyrosine kinases involved in growth (e.g., Trk). Moreover, components that control cytoskeletal dynamics (e.g., RhoGTPases and actin binding proteins) interacts with CAVs as well (Egawa et al., 2015). In addition, the presence of CAVs is thought to promote the stability of lipid-rafts (Colin et al., 2016). In particular, CAV1 is a mitochondrial ER-associated membrane (MAM)-resident protein that binds cholesterol with high affinity and is transcriptionally upregulated by cholesterol levels. CAV1 seems to promote cholesterol efflux out of the ER toward PM (Figure 3), 


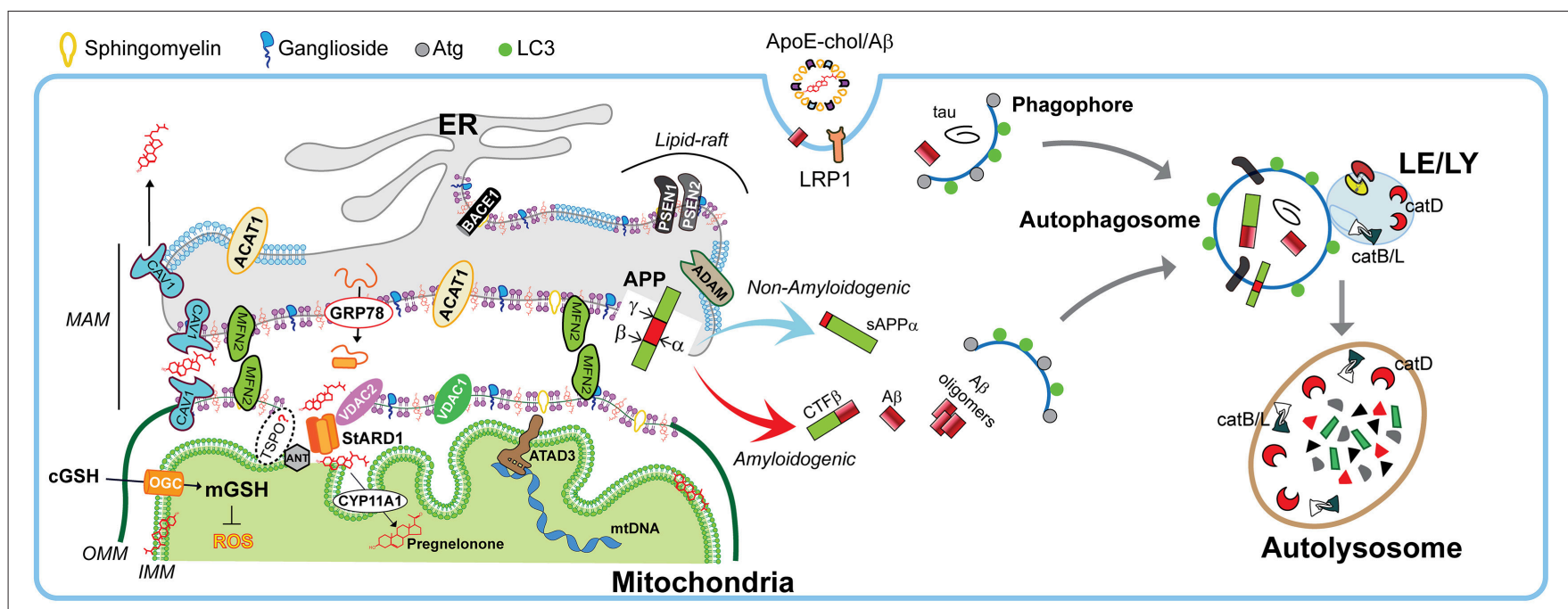

FIGURE 3 | Physiological role of MAMs in mitochondrial function and autophagy. Schematic representation of membrane-associated ER membrane (MAM) as an intracellular lipid-raft subdomain of ER (thicker region) enriched in cholesterol, anionic phospholipids (purple), sphingomyelin, and ganglosides, compared to the bulk ER (thinner region). Relevant components that connects with mitochondria both physically and biochemically are described in the text. Right panel shows the endocytic-autophagic-lysosomal system responsible to degrade and recycle proteins. In physiological conditions, the components that manage the processing of amyloid precursor protein (APP) seems to be spatially segregated in separated membrane domains (raft and non-raft, and even possibly in different neuronal organelles), which limits the amyloidogenic APP processing (red arrow) and maintain the non-amyloidogenic APP processing (light blue arrow). This segregation depends on membrane fluidity, which in turn is mediated by cholesterol levels. Moreover, the GRP78/VDAC2-dependent activation of StARD1 ensure the rapid OMM-to-IMM cholesterol delivery needed to maintain the very strict limit in the IMM-cholesterol levels that are critical to ensure the intra-mitochondrial antioxidant defense (mGSH), a proper pregnenolone production, and a correct mitochondrial DNA (mtDNA) function. Moreover, the specific integral MAM component CAV1 (light blue drawing) seems to not only modulate MAM-cholesterol contents by the cholesterol efflux out of MAM toward PM, but also increase $\alpha$-secretase activity. On the other hand, healthy neurons execute highly efficient autophagy of either intracellular tau and $A \beta$ peptides or endocytic $A \beta$. Autophagy induction begins with phagophore formation, which required LC3 and Atg proteins. The recruitment of cytosolic proteins and organelles ends when is formed the autophagosome, which fuse with lysosomes. The resulting autolysosome degrade enclosed cytosolic content by acidic hydrolases such as the neuron-enriched cathepsin D (catD) or cysteine cathepsins $B$ and $L$ (catB/L).

reducing the availability of cholesterol in MAM, hence limiting its trafficking to mitochondria (Bosch et al., 2011).

Age-dependent loss of cholesterol from PM leads to loss of lipid-rafts, impacting in decreased presynaptic vesicle fusion and disruption of neurotransmitter release. Both events are related with different forms of neurodegeneration (Egawa et al., 2015). Hence any disturbances in lipid-raft's cholesterol levels potentially contribute to pathogenesis of neurodegenerative diseases. Indeed, most of the components involved in the amyloidogenic pathway (described below), such as APP, the proteolytic enzymes BACE1 and presenilins (PSEN1/2), A $\beta$, and $\gamma$-secretase activity are enriched in lipid-rafts (Arbor et al., 2016; Colin et al., 2016), particularly in non-PM's lipid-rafts (Schon and Area-Gomez, 2013). Moreover, in physiological conditions, there are neuronal mechanisms that manage the spatial segregation of amyloidogenic machinery in separate membrane domains, and even has been suggested an organelle segregation in the neurons (Das et al., 2013). Cholesterol can regulate APP accumulation in lipid-rafts and amyloidogenesis since C99 fragment of APP (product of APP cleavage by BACE1) exhibits a binding site for cholesterol, which is immediately adjacent to $\alpha$-secretase cleavage site (K687) and the presence of cholesterol in membrane-buried GXXXG motif of C99/APP can reduce non-amyloidogenic APP processing to diminish the levels of $\alpha$-secretase-cleaved soluble $\operatorname{APP}(\operatorname{sAPP} \alpha)$, which is neurotrophic and neuroprotective (Barrett et al., 2012; Yang et al., 2014). Moreover, the $\alpha$-secretase activity that depends on membrane fluidity, is reversibly modulated by cholesterol (Yang et al., 2014), which inhibit the secretion of $\operatorname{sAPP} \alpha$ (Figure 4). This increased amiloydogenic APP processing by increased cholesterol-mediated loss of membrane fluidity and the depletion of mitochondrial GSH (mGSH) by a similar cholesterol-mediated disruption in mitochondrial membrane order accelerate neurodegeneration by increasing $A \beta$-induced cell death and oxidative stress (Barbero-Camps et al., 2014), as discussed below.

Interestingly, lipid-rafts components can undergo intracellular re-localization via ER-MAM and microtubular network allowing the presence of raft-like microdomains in mitochondrial membranes, which are enriched not only in cholesterol but also in gangliosides (although with lower content than PM), and relatively poor in phospholipids (Vance, 2014). In fact, PSEN $1 / 2$ and $\gamma$-secretase activity are localized predominantly at the MAM in concordance with the reported localizations of $A \beta$ and PSEN1 in mitochondria (Area-Gomez and Schon, 2016). This evidence supports the idea that an intracellular APP pathological processing could occur at MAM in addition to the PM (Figure 4). 


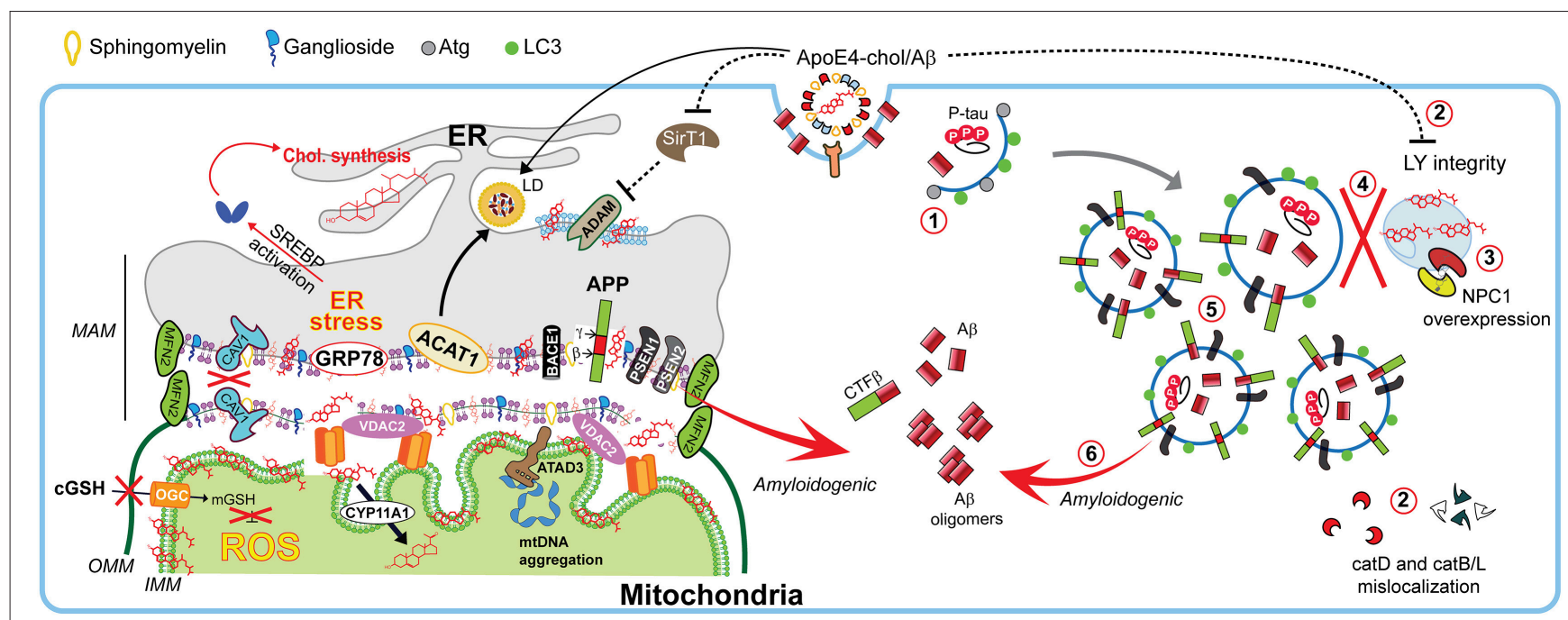

FIGURE 4 | Impact of cholesterol on MAM dynamics, mitochondrial fitness, and autophagic processes involved in Alzheimer disease. In Alzheimer disease (AD), dysregulation of cholesterol homeostasis leads to a reorganization of ER-membrane domains and co-locating the amyloidogenic components at the MAM to increase $A \beta$ biogenesis. Schematic representation of the endocytic-autophagic-lysosomal axis involved in AD, which includes: (1) decreased expression and activity of autophagy-inducing proteins such as Atg; (2) ApoE4-induced disruption of lysosomal integrity and mislocalization of lysosome proteases; (3) increased NPC1 overexpression in disease-affected brain areas; (4) defective lysosomal fusion, resulting in concomitant (5) high levels of autophagic and endosomes vacuoles containing undigested pathological proteins such as PSEN1 and APP capable of (6) generating A $\beta$ peptides.

\section{Mitochondrial Cholesterol Trafficking}

Given the fact that the ER makes extensive MCS with other organelles such as PM, Golgi, and mitochondria, it is likely that cholesterol can move from ER to these downstream destinations. Indeed, the ER-subdomain of MAMs physically interact with outer mitochondrial membrane (OMM), defining a specific suborganelle characterized by unique biochemical properties and specialized functions that involve both organelles (Figure 3). These actions are dependent on specific biophysical properties of MAM, which are determined by the dynamics of protein/lipid composition, and functional alterations in this particular interface are associated with a number of neurodegenerative disorders (see below). Among others, MAM have been shown to participate in multiple pathways, including calcium homeostasis (e.g., IP3 receptors); phospholipid metabolism (e.g., phosphatidylserine synthase 2, PSS2); ER stress (e.g., GRP78), and cholesterol metabolism (e.g., ACAT1 and HMGCR; Vance, 2014; Area-Gomez and Schon, 2016, 2017; van Vliet and Agostinis, 2017). Interestingly, in vitro reconstitution assays showed that among ER membranes, MAMs have higher cholesterol content, which is relevant to the association between ER and mitochondria (Area-Gomez et al., 2012). Furthermore, mitochondrial-related proteins such as adenine nucleotide transporter (ANT) at inner mitochondrial membrane (IMM) and voltage-dependent anion channel (VDAC) at OMM have also been found in MAM, supporting the idea that cholesterol could move from ER to mitochondria via a TSPO/VDAC/STARD1/ANT-dependent mechanism (Figure 3). This is based on observations indicating an interaction between ANT, VDAC, and TSPO (Allouche et al., 2012; Vance, 2014; Stocco et al., 2017), and the fact that STARD1 is first loaded onto the MAM to interact with VDAC2 for its processing to mature active form at the OMM (Prasad et al., 2015). However, the role of TSPO in mitochondrial cholesterol trafficking is under debate. Recent evidence using genetic models, such as loxP-Cre-based global TSPO deletion (Tspo-/-) or Leydig cellspecific TSPO conditional knockout (Tspo ${ }^{\mathrm{c} \Delta / \Delta}$; Morohaku et al., 2014), and CRISP/Cas9-based deletion of TSPO in the MA-10 mouse Leydig cell line (MA-10 ${ }^{T s p o s} / \Delta$; Tu et al., 2015) have shown a minimal effect of TSPO ablation on steroidogenesis. Most surprisingly, it was determined that human adrenal H295R cell line did not express TSPO, even though these cells are competent in the synthesis of steroids (Tu et al., 2014). Since the expression of TSPO in STAR-deficient mice is unable to compensate the mitochondrial cholesterol transport deficit (Caron et al., 1997; Ishii et al., 2012) and that isolated Tspo-deficient mitochondria exhibit a normal mitochondrial cholesterol import (Banati et al., 2014), it is conceivable that TSPO plays a minor role in mitochondrial cholesterol import for steroidogenesis, questioning the TSPO-STARD1 interaction (Selvaraj and Tu, 2016).

Among subcellular membranes, mitochondrial membranes and particularly the IMM, are cholesterol-poor. However, cholesterol must reach at IMM for the synthesis of neurosteroids in the brain, which are necessary for normal stimulation of GABAergic currents, Purkinje cells's response modulation to excitatory amino acids and the enhancement of memory function (Stocco et al., 2017). StAR-dependent delivery of cholesterol from the OMM to the IMM governed by STARD1 is the ratelimiting step for steroidogenesis by facilitating generation of pregnenolone (precursor of all steroids) from cholesterol through metabolism by cytochrome p450scc (CYP11A1, Figure 3; 
Elustondo et al., 2017; Stocco et al., 2017). In steroidogenic cells (including specialized neurons), the very low IMM-cholesterol levels enable a faster modulation of steroidogenesis by the rate of the OMM-to-IMM transport of cholesterol rather than enzymatic activity-dependent pathways. However, this process implies that even small changes in cholesterol levels can strongly modify the biophysical/functional features of IMM, which sensitizes mitochondria to alterations in cholesterol content (Elustondo et al., 2017).

Besides ER-derived cholesterol import, LE/LY-derived cholesterol also can be imported into mitochondria in a process involving another START domain containing transport protein, STARD3 (also known as MLN64 and component of ER-LE MCS; Figure 2; Charman et al., 2010). STARD3 N-terminal domain is anchored in the LE membrane (Alpy and Tomasetto, 2006) and its cholesterol-binding START C-terminal domain facing the cytosol (Alpy et al., 2001). Interestingly, STARD3 is present in LE containing cholesterol transporter ABCA3, which can recycle cholesterol to the PM and other membranes (van der Kant et al., 2013). Recently, Balboa and coworkers have reported that overexpression of STARD3 in both normal and NPC1-deficient hepatic cells, increases mitochondrial cholesterol content, resulting in $\mathrm{mGSH}$ depletion and mitochondrial dysfunction (Balboa et al., 2017).

In addition, emerging evidence points to the existence of a close relation between ER and lipid droplets, and lipid droplet-derived cholesterol could traffic to mitochondria through the ER (Barbosa and Siniossoglou, 2017). Moreover, recently it has been proposed that soluble $\mathrm{N}$-ethylmaleimide sensitive factor attachment receptor (SNARE) proteins can facilitate the interaction of lipid droplets and mitochondria (Lin et al., 2016). Thus, LE/LY and ER seem to act as the source of mitochondrial cholesterol, although the specific contribution of cholesterol originating from each organelle is not known.

\section{Cholesterol and Mitochondrial DNA}

Moreover, in addition to the regulation of membrane physical properties, cholesterol content in the IMM can be relevant for mitochondrial DNA (mtDNA) organization. A proper mitochondrial DNA (mtDNA) transcription/replication is ensure by its association with protein forming complexes called nucleoids, which include the mtDNA polymerase polG and the mtDNA helicase Twinkle. Both proteins are part of a membrane-associated platform. Recent findings have indicated that this platform is associated with cholesterol-enriched membrane structure in close association with ER (Figure 3; Gerhold et al., 2015). Therefore, as cholesterol co-sediments with mtDNA, hence it is conceivable that perturbed cholesterol homeostasis could disrupt mtDNA replication. Indeed, recent findings using human fibroblasts from patients with deletions at ATPase family, AAA domain-containing protein 3 (ATAD3, a raft-like domain resident of MAM and an enhancer of hormonal-induced steroidogenesis) described localized mtDNA aggregation, which translated in impaired distribution and slowed down mtDNA synthesis (Desai et al., 2017). Interestingly, treatment of normal fibroblasts with inhibitors of cholesterol synthesis, trafficking (pravastatin, U18666A) or with soluble cholesterol induced similar features resembling the ATAD3lacking phenotype. Moreover, similar observations were found in fibroblasts from patients with NPC disease (discussed below; Desai et al., 2017), suggesting that a strict limit in the IMMcholesterol levels is necessary to ensure correct mtDNA function. Thus, disruption in mitochondrial cholesterol trafficking and distribution can have far-more reaching consequences other than altering intramitochondrial antioxidant defense mechanism and physical properties, contributing to mitochondrial dysfunction through impaired generation of mtDNA-encoded subunits of the respiratory chain complexes, which impact in cell physiology, energetics and survival, culminating in neurodegeneration.

\section{ROLE OF INTRACELLULAR CHOLESTEROL IN NEURODEGENERATION}

\section{The Ups and Downs of Cholesterol in Neurodegeneration: The Case of Alzheimer Disease}

Given the key role of cholesterol in the regulation of membrane biophysical properties and cell functions through modulation of signaling pathways, alterations in cholesterol homeostasis have been associated with the disruption of brain functions and onset of major neurodegenerative diseases (for more details see Tables 1-3). However, while there has been cumulative data linking increased total cholesterol levels with neurodegeneration, there is also evidence indicating the association for decreased total cholesterol levels with neurodegenerative disorders. This conundrum is well-illustrated in the case $\mathrm{AD}$ and although alterations in cholesterol homeostasis have been linked to neurodegenerative disease, however, in $\mathrm{AD}$ whether total cholesterol levels are upregulated or downregulated is controversial (Table 1). As briefly mentioned above, amyloidogenic APP processing into toxic A $\beta$ peptides occurs in the cholesterol-enriched lipid-rafts domains of the PM. One of the first lines of evidence that brain's $A \beta$ production can be influenced by cholesterol came from observations pointed that cholesterol-enriched diet is able to increase the amyloidosis in rabbit hippocampal neurons (Sparks et al., 1994). Subsequent data from transgenic mice model designed to deposit cerebral $\mathrm{A} \beta$ showed that diet-induced hypercholesterolemia leads to increment in both $\mathrm{A} \beta$ deposition and amyloid plaque formation (Refolo et al., 2000), in line with findings in mice fed diets enriched in cholesterol (Shie et al., 2002; Thirumangalakudi et al., 2008). Although these observations linking dietary cholesterol intake to $\mathrm{AD}$ are puzzling in view of the negligible rate of cholesterol transport from the periphery into the brain due to intact $\mathrm{BBB}$, it has been shown that $\mathrm{BBB}$ permeability is impaired in $\mathrm{AD}$ (Ujiie et al., 2003). Consistent with the role of cholesterol in $\mathrm{AD}$ pathogenesis, hypercholesterolemia not only correlates with $\mathrm{A} \beta$ deposition, but also is a risk factor for $\mathrm{AD}$, while statins intake confers a lower incidence of AD (Notkola et al., 1998; Wolozin et al., 2000; Martins et al., 2009). Moreover, several genes that encodes to protein-related cholesterol homeostasis, 


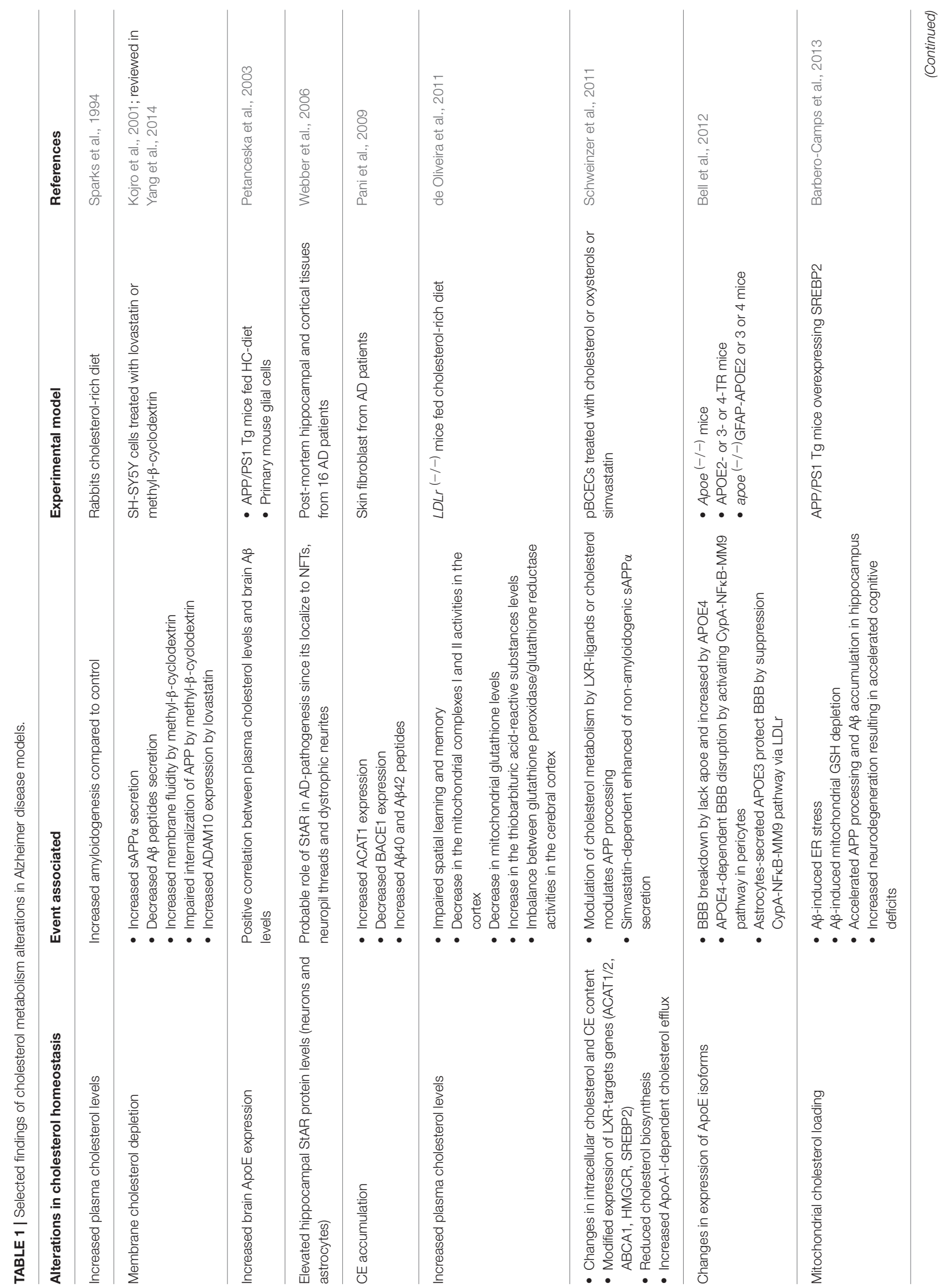




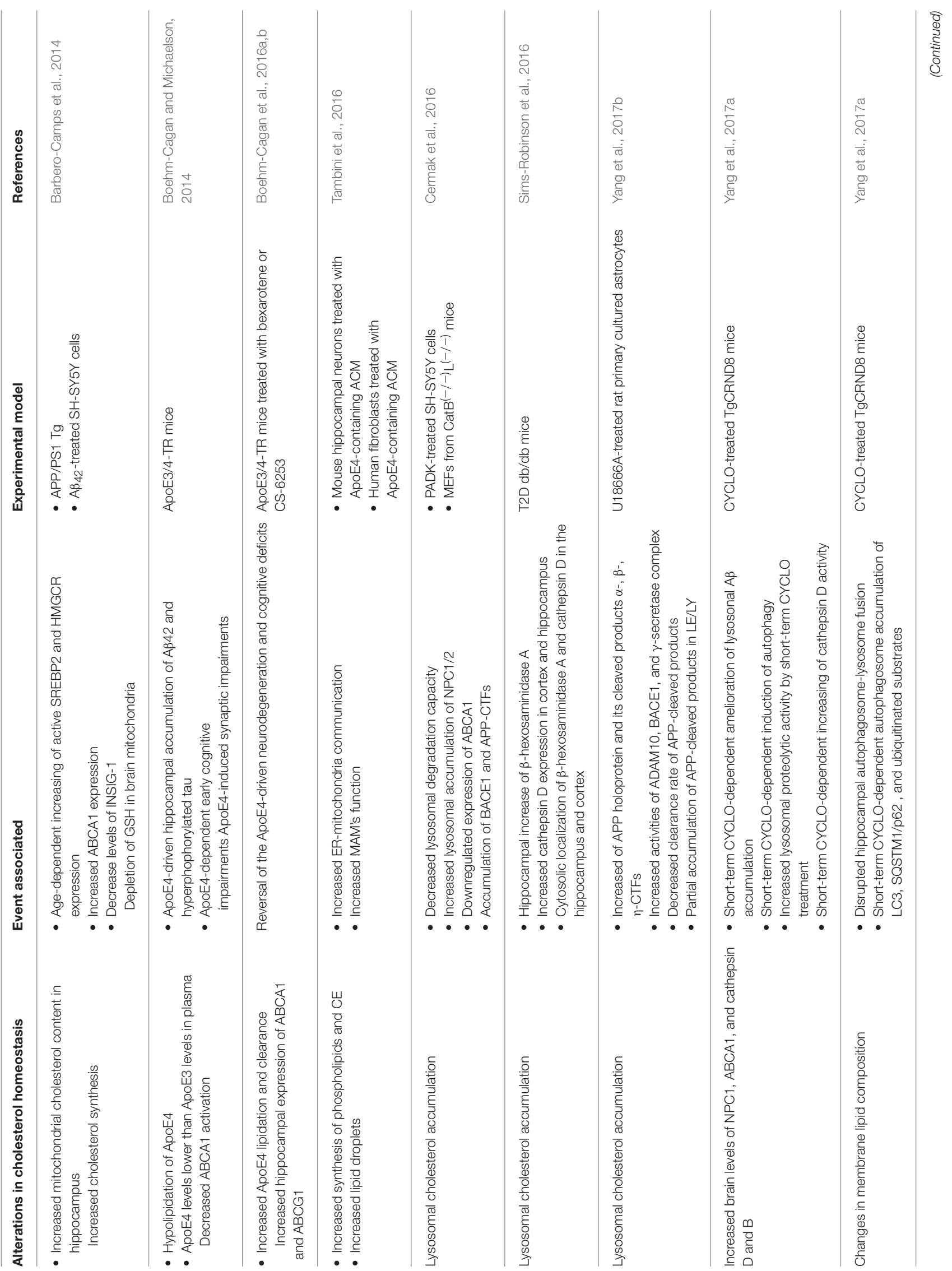




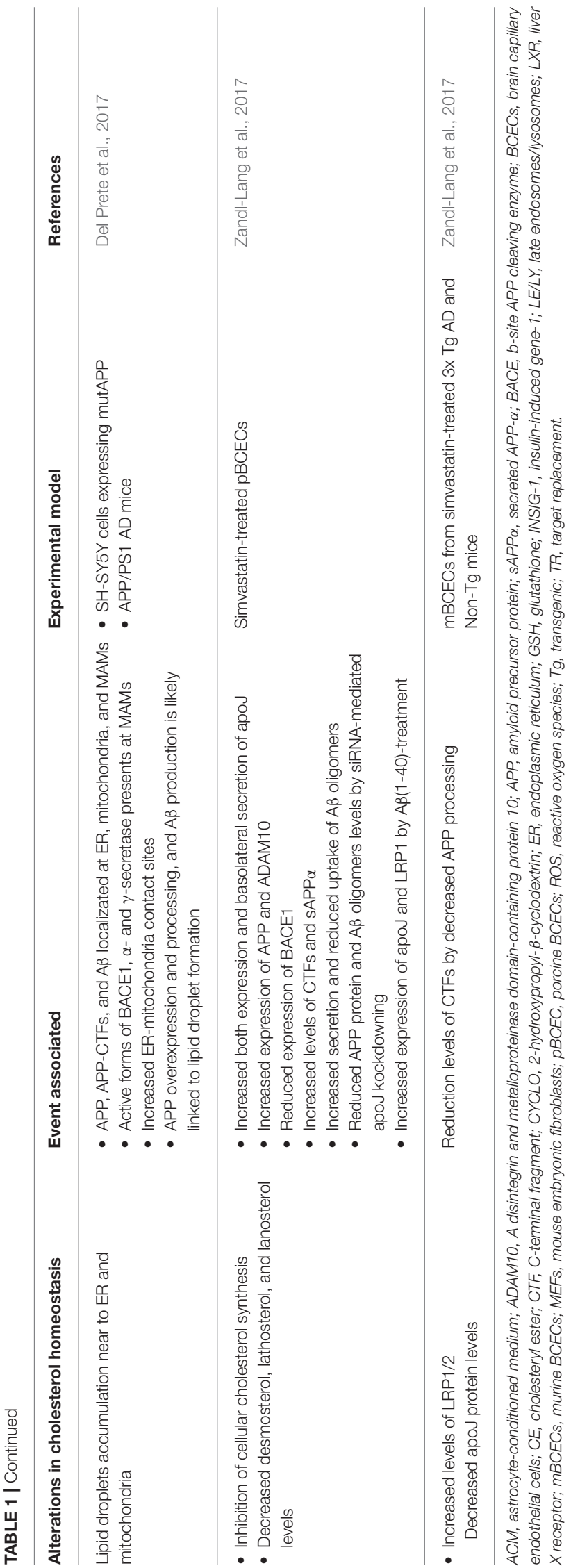

including ApoE, CYP46A1, ACAT1/SOAT1, the cholesterol efflux transporters ABCA1 and ABCA7, and LRP1 have been linked to the risk, development or progression of $\mathrm{AD}$ (Chen et al., 2008; Kim et al., 2009; Di Paolo and Kim, 2011). Intriguingly, despite these findings for the association of increased cholesterol to $\mathrm{AD}$, there is evidence that decreased cholesterol levels are associated with and promote AD. For instance, previous studies showed a correlation between AD-brain cholesterol content and the reduction of fluidity on the hydrocarbon core region of hippocampal membranes of $\mathrm{AD}$-patients; moreover, respect to controls, the temporal gyrus but not the cerebellum of $\mathrm{AD}$ patients showed a reduction of both cholesterol/phospholipids mole ratio and total cholesterol levels (Mason et al., 1992; Eckert et al., 2000). In addition, in comparison with control subjects decreasing of 24-OHC levels in the frontal and occipital cortex of $\mathrm{AD}$-patients have been reported with an increment of mevalonate pathway's inhibition rather than decreased production of $A \beta$ and amyloid plaques (Heverin et al., 2004; Cole et al., 2005). Furthermore, in the context of examining the homeostasis of lysosomal cholesterol through NPC1, Kagedal and co-workers described decreased cholesterol levels in the hippocampus but not cerebellum of patients with $\mathrm{AD}$ compared to control subjects (Kagedal et al., 2010). Thus, these findings illustrate the lack of correlation between total cholesterol levels in brain and $\mathrm{AD}$.

Besides $\mathrm{AD}$, homeostasis disturbance in the cholesterol biosynthesis has been also associated with rare hereditary diseases. Indeed, early life impairment brain function is associated to mutations of genes related with cholesterol synthesis. For instance, mutations in DHCR7 within the Kandutsh-Russell pathway (Figure 1) result in Smith-LemliOpitz syndrome (SLOS), a disease characterized by low cholesterol levels in the brain that primarily cause intellectual disabilities, psychiatric problems and delayed maturation of both motor and language skills (Segatto et al., 2014). On the other hand, dysfunction of last step of the astrocytespredominant Bloch pathway (i.e., enzyme-mediated reduction of desmosterol to cholesterol) by mutations in DHCR24 gene results in desmosterolosis (Figure 1), also characterized by low brain's cholesterol content related with a generalized malformation of brain's structures, such as ventricular enlargement, thinning of white matter and seizures, defective corpus callosum, microcephalia, and hydrocephalia (Schaaf et al., 2011). Whether this disease is causally linked to reduced cholesterol levels or increased desmosterol remains to be established. In addition, other neurological diseases associated with disturbance of cholesterol biosynthesis include the Rett syndrome (RS), a neurological disorder caused by mutations in the X-linked MECP2 gene with clinical features that include defects in motor control, cognitive skills, and social interactions such as intellectual incapacities, and autistic behavior. RS has been related with changes in cholesterol metabolism since HMGR, squalene epoxidase, and CYP46A1 are altered in Mecp-2 knock-out mice in an age-dependent manner (Martin et al., 2014). In the following sections we will briefly summarize the role of intracellular cholesterol pools, in neurodegeneration. 


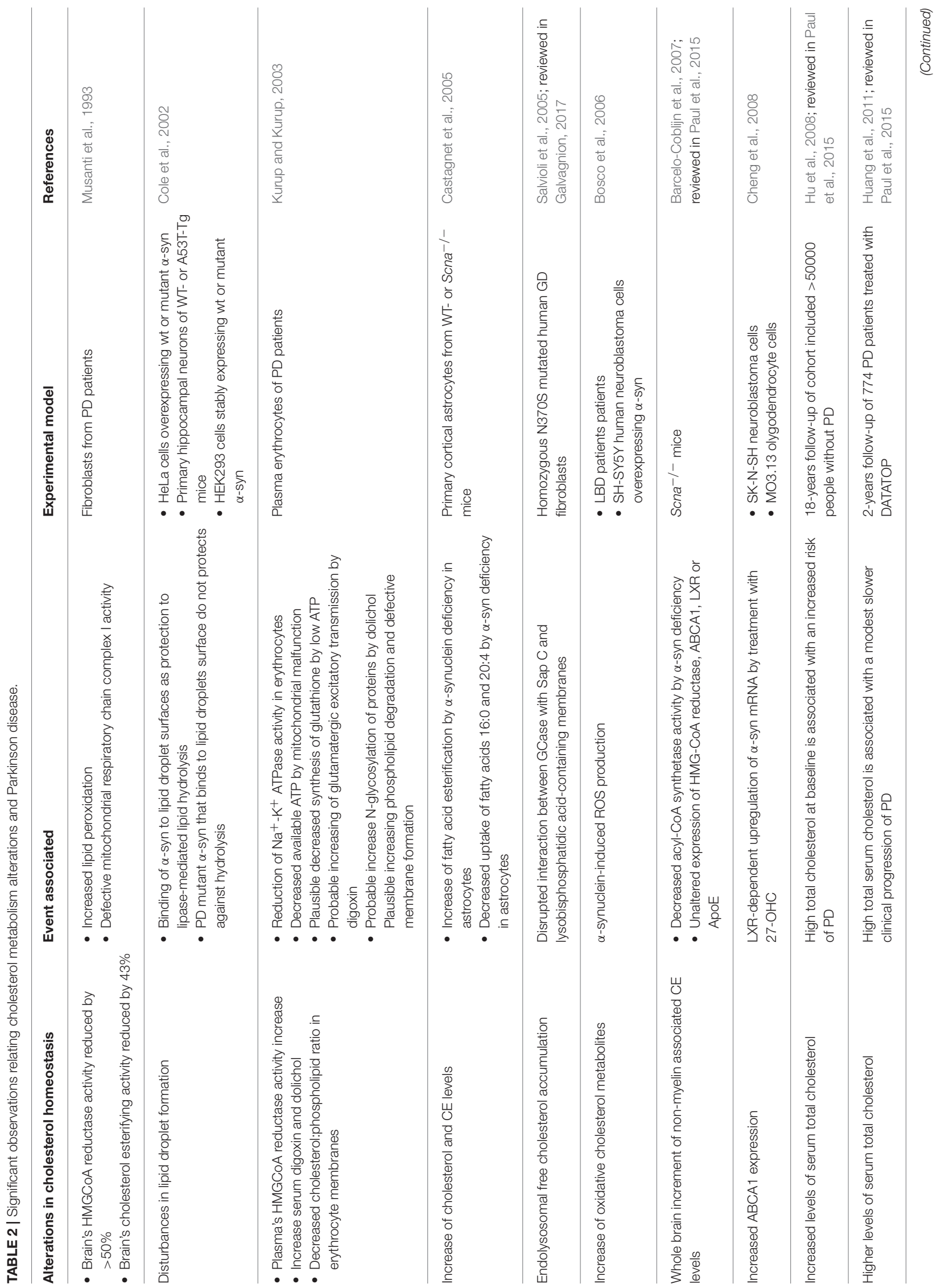




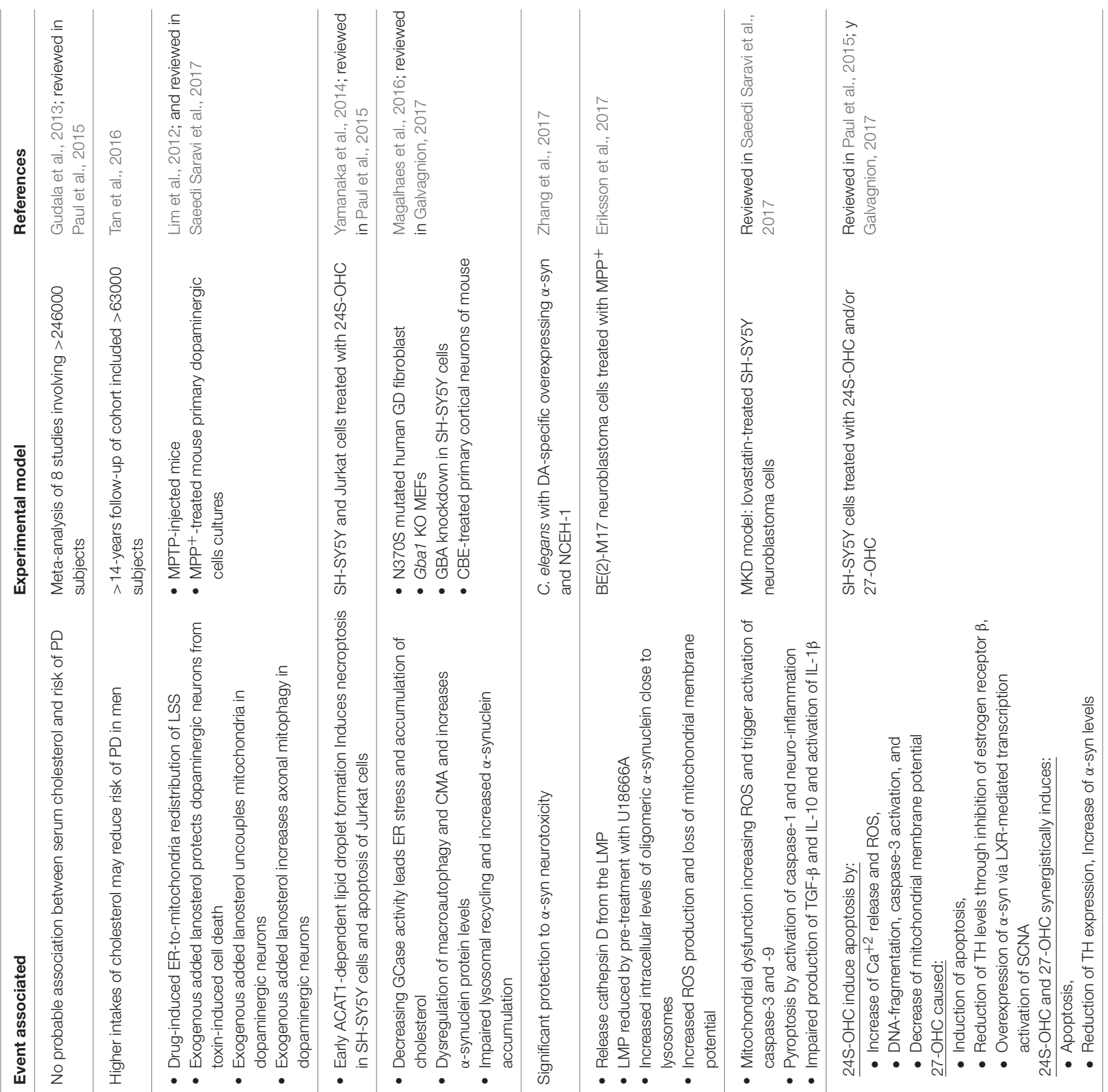

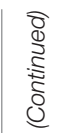

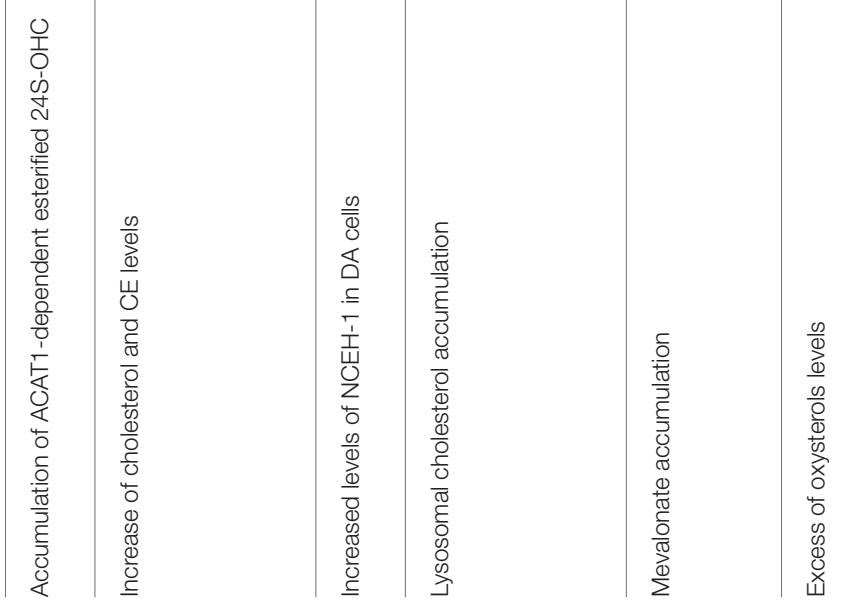




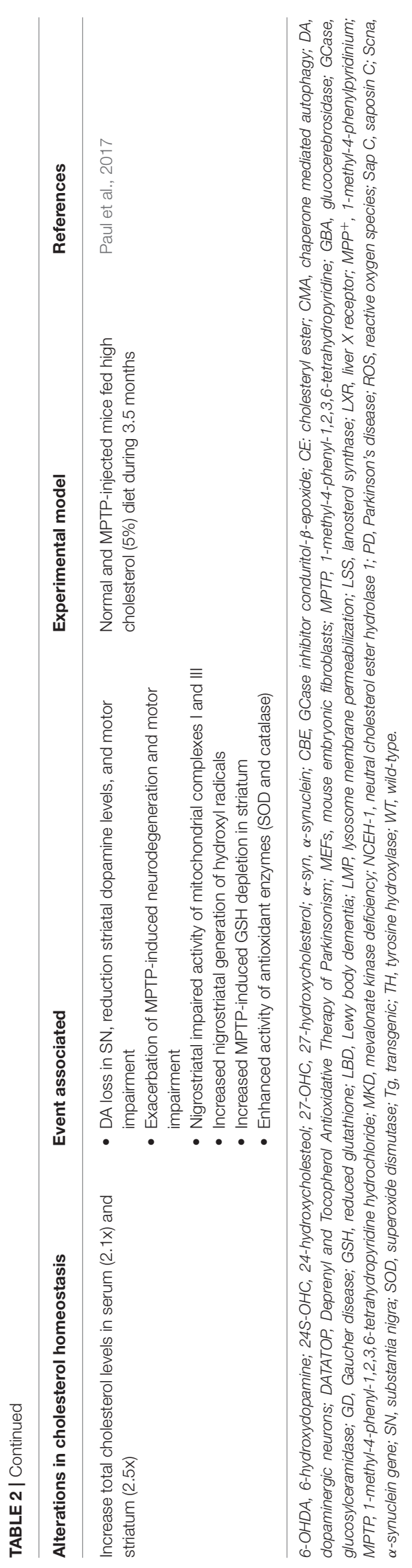

\section{Lysosomal Cholesterol in Niemann Pick Type C Disease, Alzheimer Disease, and Parkinson Disease}

Consistent with its key role in intracellular cholesterol trafficking, alterations in the molecular machinery involved in the release of cholesterol from LE/LY have an impact in brain pathophysiology. For instance, Rab8 GTPase, which promotes the LE/LY to PM cholesterol efflux via LRO (Figure 2) and participates in polarized neurite growth and in the dynamics of glutamate receptors in postsynaptic compartment ( $\mathrm{Ng}$ and Tang, 2008), has been involved in $\alpha$-synuclein aggregation and reduced toxicity in cellular models for PD (Yin et al., 2014). In addition, alterations in release of the LE/LY cholesterol by mutations in NPC1/2 proteins cause Niemann-Pick type C (NPC) disease (Figure 2), a progressive and fatal visceral, neurological and psychiatric disorder characterized by increased accumulation of unesterified free cholesterol and other lipids (e.g., glycosphingolipids) predominantly in LE/LY in NPC patient fibroblasts and in axons of cultured neurons with reduced cholesterol levels in the PM. Besides brain pathology, some patients with NPC disease exhibit liver disease and hepatosplenomegaly and like neurons, hepatocytes from NPC null mice exhibit increased cholesterol accumulation and sphingolipids (Mari et al., 2006; Torres et al., 2017). Purkinje cells in the cerebellum are the most vulnerable neurons in NPC disease, accounting for the characteristic ataxia of NPC patients (Malnar et al., 2014). Moreover, not only synaptic vesicle composition and morphology are affected, but also endosomal organelle transport, leading to neuronal loss in NPC disease (Schultz et al., 2016). Animal models for NPC disease (i.e., NPC knockout mice) mimic most of the pathological features of NPC patients and display loss of neurons rather than astrocytes as the key determinant of the neurodegeneration in NPC disease (Schultz et al., 2016). Moreover, increased levels of cholesterol in mitochondria from brain and hepatocytes of $\mathrm{NpC1}^{-/-}$mice have been reported as well (Yu et al., 2005; Fernandez et al., 2009), highlighting the close relationship between both organelles in cholesterol trafficking. Although there are considerable neuropathological differences at the anatomical level between NPC and AD, both diseases share many parallels, including progressive neurodegeneration, LE/LY abnormalities, cholesterol accumulation, hyperphosphorylation of tau, APP processing and $A \beta$ accumulation (Figure 4). While there is no evidence of amyloid plaques in human NPC brains, likely due to premature death, several studies with NPC models in cells and mice showed that the amyloidogenic APP processing can be modulated by the disruption of normal cholesterol trafficking observed in NPC (Malnar et al., 2014). Indeed, compared to CHO- $w t$ cells, $\mathrm{CHO}-\mathrm{NPC}^{-} /^{-}$cells exhibit increased levels of BACE1-generated APP metabolites ( $\mathrm{APP} \beta$ and C99) and an intracellular $A \beta$ accumulation, which was reversed by cholesterol depletion, indicating that the enhanced BACE1 cleavage of APP occurs as a consequence of cholesterol accumulation rather than NPC1 loss (Malnar et al., 2010). Although expression of NPC1 has been poorly characterized in AD, Kagedal and coworkers described in the hippocampus and frontal cortex of $\mathrm{AD}$ an increased expression of NPC1 both at the mRNA and 


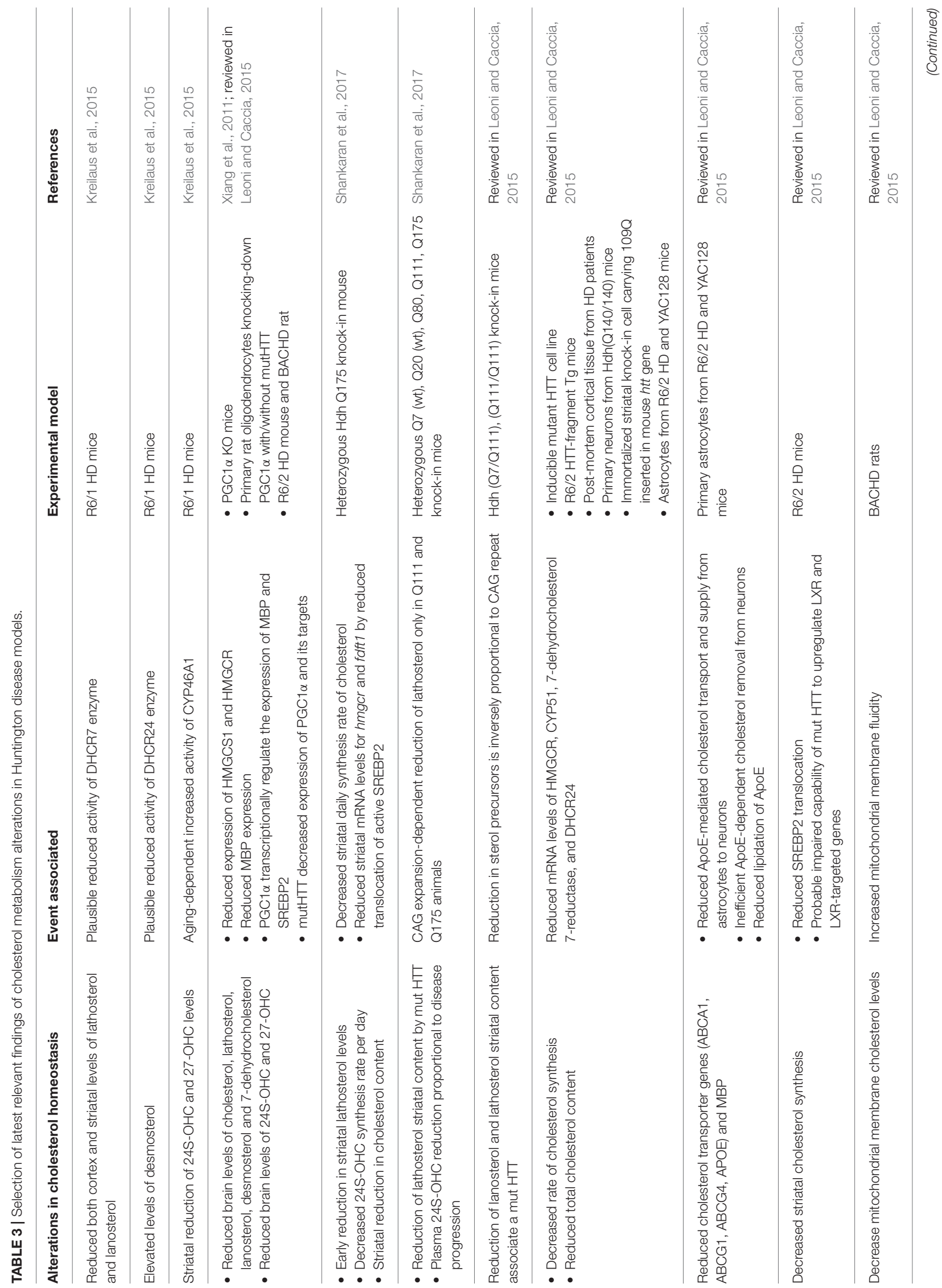


protein levels compared to control subjects, with no changes in cerebellum (Kagedal et al., 2010). Similarly, 12-month-old APP/PS1 transgenic mice showed increased hippocampal murine NPC1 transcripts compared to age-matched wild type mice (Kagedal et al., 2010). Thus, these findings imply the impossibility of accumulation of lysosomal cholesterol in $\mathrm{AD}$, and suggest that the lysosomal deficiency that contributes to impair $A \beta$ degradation in $\mathrm{AD}$ possibly occur by mechanisms independent of lysosomal cholesterol accumulation. Among the parallels existing between NPC disease and AD, the mitochondrial cholesterol loading is the most common link between these diseases rather than lysosomal cholesterol accumulation (Webber et al., 2006; Fernandez et al., 2009; Torres et al., 2017).

To assess the impact of lysosomal cholesterol accumulation in APP processing, $\mathrm{A} \beta$ generation and $\mathrm{AD}$ pathology, Maulik et al. generated a mouse line (ANPC), which expressed mutant human APP in the context of genetic deletion of NPC1 (Maulik et al., 2015). The phenotype included increased cholesterol accumulation, leading to enhanced APP C-terminal $\mathrm{CTF}$ fragments and $\mathrm{A} \beta$ generation, as well as decreased clearance of peptides and increased $\gamma$-secretase activity. Cholesterol sequestration in ANPC mice impaired endocytic-autophagiclysosomal but not proteasome clearance of APP-CTFs/A $\beta$ peptides, and vulnerable brain regions of ANPC mice exhibited increased oxidative stress and susceptibility to $\mathrm{H}_{2} \mathrm{O}_{2}$-induced toxicity. In line with these effects of increased cholesterol in lysosomes, extraction with 2-hydroxypropyl-cyclodextrin (CDX) in $\mathrm{AD}$ models revealed a dual effect of $\mathrm{CDX}$ in modulating the mechanisms involved in AD pathology (Yang et al., 2017a). While CDX enhanced lysosomal activity, it impaired autophagosomelysosome fusion resulting in defective autophagy and clearance of $\mathrm{A} \beta$ fragments (Yang et al., 2017b). Together, these findings strongly suggest that unlike NPC disease, the accumulation of cholesterol in lysosomes does not seem to be a characteristic feature of $\mathrm{AD}$ and that prolonged treatment with $\mathrm{CDX}$ should be carefully evaluated to avoid unwanted effects on autophagy. Whether the increase in NPC1 in AD is a compensatory mechanism to stimulate lysosomal cholesterol efflux remains to be determined.

On the other hand, metabolic disorders such as type 2 diabetes mellitus (T2DM), which is a risk factor for $\mathrm{AD}$, are associated with dementia involving several pathophysiological mechanisms (Li and Huang, 2016). For instance, oxidized cholesterol by intrinsic T2DM hypercholesterolemia in $\mathrm{db} / \mathrm{db}$ mice not only leads to destabilization of lysosomes, but also alterations in the expression and activity in the hippocampus of the lysosomal enzymes, such as cathepsin D (CatD; Figure 4; Sims-Robinson et al., 2016) by an ApoE4-dependent mechanism related to disruption of lysosomal integrity, including the mislocalization of CatD, which contributes to degradation of the antioxidant protein thioredoxin-1 (Persson et al., 2017). However, some authors pointed to cysteine cathepsins B and L (CatB/L; Figure 4) as the main lysosomal enzyme(s) involved in the abnormalities of intracellular cholesterol trafficking related to AD-like features, since unlike CatD, the CatB/L inhibition in SH-SY5Y human neuroblastoma cells by PADK-treatment or its genetic ablation in $\mathrm{CatB}^{(-/-)} / \mathrm{L}^{(-/-)}$MEFs resembled NPC disease features 
(e.g., lysosomal dysfunction by accumulation of both cholesterol and NPC1; and downregulation of ABCA1 expression) and promotes amyloidogenesis assessed by BACE1 and APP-CTFs accumulation (Cermak et al., 2016). Nonetheless, it should be noted that CatD mediates the proteolysis of ApoE and both colocalized in $\mathrm{AD}$ frontal cortex on neuritic plaques, which in despite above, suggests a plausible role of CatD in the $\mathrm{AD}$ pathogenesis through ApoE fragments (Zhou et al., 2006). In fact, recently it has been described that ApoE4 is able to translocate to the nucleus and even acting as a transcription factor given its capability to bind to double-stranded DNA with high affinity (infra nanomolar; Theendakara et al., 2016). For instance, Rao and colleagues reported that ApoE4 specifically represses the expression of Sirtuin 1 (SirT1), a transcriptional activator of the $\alpha$-secretase ADAM10 that promotes the production of the neuroprotective sAPP $\alpha$ peptide, hence contributing to stimulated $\mathrm{A} \beta$ production (Figure 4; Theendakara et al., 2016).

$\mathrm{PD}$ is the second most common neurodegenerative disorder after $\mathrm{AD}$ and it is characterized by the accumulation of intraneuronal cytoplasmic inclusions of Lewy bodies, caused by the aggregation of $\alpha$-synuclein. Similar to $\mathrm{AD}$, there is a large body of data linking alterations in total cholesterol levels with neurodegeneration in PD (Table 2). In cellular models of $\mathrm{PD}$, the neurotoxin 1-methyl-4-methylpyridinium $\left(\mathrm{MPP}^{+}\right)$, a mitochondrial toxin, triggered lysosomal cholesterol accumulation preceding cell death (Eriksson et al., 2017). This event was ameliorated by preincubation with U18666A, suggesting that neurons respond to early apoptotic stress increasing accumulation of lysosomal cholesterol to preserve the integrity of lysosomal membrane. Moreover, high cholesterol loading also promotes $\alpha$-synuclein accumulation, and the treatment with lovastatin reduced $\mathrm{MPP}^{+}$-induced cell death by decreasing ROS but did not prevent lysosomal cholesterol increased nor affected $\alpha$-synuclein accumulation. Thus, these findings indicate a dual role of cholesterol accumulation in $\mathrm{PD}$, protecting against lysosomal membrane permeabilization and cell death, while stimulating the accumulation of $\alpha$ synuclein. Whether cholesterol accumulates in lysosomes or if the expression of proteins regulating lysosomal cholesterol trafficking is altered in patients with $\mathrm{PD}$ remains to be investigated.

\section{Mitochondrial Cholesterol in Alzheimer Disease, NPC Disease, and Huntington Disease}

Mitochondria are cholesterol-poor organelles compared to PM. However, the restricted pool of mitochondrial cholesterol, particularly at IMM, is crucial for the synthesis of neurosteroids in the brain and for physiological GABAergic responses that modulate memory function (Stocco et al., 2017). As mentioned above, the rate of cholesterol translocation from OMM to IMM controls steroidogenesis, indicating that changes in the levels of cholesterol in IMM has a significant impact in the extramitochondrial functions in specialized tissues mediated by the generation of pregnenolone and subsequent steroids derived from the metabolism of cholesterol in the IMM.
Moreover, pathological conditions leading to unphysiological cholesterol accumulation in mitochondrial membranes can have a profound effect in mitochondrial function, including defective mitochondrial antioxidant defense. Indeed, we have recently provided evidence for a critical role of mitochondrial cholesterol loading in the aggravation and acceleration of AD through the generation of a novel genetic mouse model of $\mathrm{AD}$ characterized by the overexpression of SREBP-2 in the background of APP/PS1 transgenic (APP/PS1/SREBP2; Barbero-Camps et al., 2013). The pathological events in APP/PS1/SREBP2 mice precipitating ADlike symptoms were dependent on mGSH depletion (Figure 4), since in vivo replenishment of $\mathrm{mGSH}$ with cell-permeable GSH monoethyl ester (GSHee) attenuated neuropathological features of AD in APP/PS1/SREBP-2 mice, including decreased neuroinflammation, cell death and tau-phosphorylation, which led to improvement of cognitive defects (Barbero-Camps et al., 2013). Quite intriguingly, although NPC disease is primarily characterized by increased lysosomal cholesterol, NPC fibroblasts, and $N p 1^{-/-}$mice also exhibit increased mitochondrial cholesterol accumulation and subsequent mGSH depletion (Torres et al., 2017). Similar to the APP/PS1/SREBP2 $\mathrm{AD}$ model, $\mathrm{NPC1}^{-/-}$mice display increased expression of StARD1 by an ER stress-independent mechanism, which remains to be uncovered (Torres et al., 2017). Treatment with GSHee restored $\mathrm{mGSH}$ levels in brain of $\mathrm{NPC1}^{-/-}$mice and fibroblasts from NPC patients without alterations in the profile of most sphingolipids, resulting in increased life span, improved mitochondrial function and enhanced Purkinje cell survival, underlying the reversal of locomotor deficits. Interestingly, these beneficial effects of GSHee contrast with the failure of $\mathrm{N}$ acetylcysteine (NAC) to improve NPC pathology due to its inability to restore mGSH levels despite significant increase in cytosol GSH pool (Torres et al., 2017). The levels of mitochondrial reactive oxygen species (mROS) are modulated by $\mathrm{mGSH}$ and increased mROS levels contribute to mDNA mutations, mitochondrial dysfunction, and cellular death (Ribas et al., 2014). The mitochondrial transport of GSH from cytosol where it is synthesized de novo from its constituent aminoacids is dependent on mitochondrial membrane composition and fluidity (Ribas et al., 2014) and accumulation of cholesterol in IMM governed by StARD1 affects biophysical membrane properties, function and antioxidant defense, which overall contribute to the pathology of $\mathrm{AD}$ (Figure 4). These findings are in line with the central role of changes in membrane biophysical properties in AD (Yang et al., 2014). Indeed, an increase in membrane fluidity correlates with a rearrangement of cholesterol, sphingomyelin, and proteins-related to APP processing between raft and non-raft domains, shifting APP processing to non-amyloidogenic pathway enhancing $\operatorname{sAPP} \alpha$ production (Yang et al., 2014). Moreover, CDX-mediated removal of cholesterol increased membrane fluidity and leads to $\alpha$-secretase overexpression in neuroblastoma SH-SY5Y cells (Kojro et al., 2001), whereas, higher levels of mitochondrial APP levels in AD-affected brain areas have been found to correlate with mitochondrial dysfunction (Lin and Beal, 2006).

The role of StARD1 in human $\mathrm{AD}$ is poorly understood. Increased StARD1 expression in pyramidal hippocampal 
neurons of $\mathrm{AD}$-patients has been described (Webber et al., 2006). Although the functional consequences of this event in the regulation of mitochondrial cholesterol levels and mitochondrial function were not examined in human $\mathrm{AD}$, enhanced mitochondrial cholesterol loading in $\mathrm{AD}$ mouse models has been shown to sensitize neurons to $A \beta$-induced inflammation and toxicity by depleting mGSH through a mechanism dependent of ER stress, which in turn modulates lipid metabolism (Figure 4; Barbero-Camps et al., 2014). In addition, $A \beta$-induced ER stress is indirectly involved as an effector of the $\mathrm{A} \beta$-neurotoxicity in early stages of AD (Hoozemans et al., 2012). In fact, treatment with ER stress inhibitors such as TUDCA blocked $\mathrm{A} \beta$-induced mGSH depletion (Barbero-Camps et al., 2014) and the immunoreactivity of the ER stress marker GRP78 correlated with Braak AD-stages in postmortem AD-brains, which also exhibit alterations in proteins levels of the ER stress components (Placido et al., 2014). In line with recent findings in mouse hepatocytes showing that ER stress induces the expression of StARD1 (Fernandez et al., 2013), it is conceivable that $A \beta$ mediated ER stress triggers mitochondrial cholesterol loading through StARD1 upregulation, which in turn sensitizes to $A \beta$ induced cytotoxicity by $\mathrm{mGSH}$ depletion due to mitochondrial cholesterol accumulation. This link between A $\beta$-ER stressStARD1-mitochondrial cholesterol loading-mGSH depletion can be abrogated by chaperones that block ER stress. Overall, while cholesterol may modulate APP amiloydogenic processing and $A \beta$ production, the mitochondrial cholesterol pool emerges as a novel factor in modulating the susceptibility to the noxious effects of toxic $A \beta$ fragments and tau-phosphorylation underlying $\mathrm{AD}$. Due to the restriction of efficient cytosol GSH transport to mitochondrial imposed by mitochondrial-mediated decrease in membrane dynamics, strategies that increase cytosol GSH levels may not be sufficient to boost mGSH levels. Novel GSH precursors, such as $\gamma$-glutamylcysteine ethyl ester $(\gamma$ GCEE) or S-acyl GSH thioester have been shown to increase total GSH in fibroblasts from patients with $\mathrm{AD}$ and protect primary neurons against $\mathrm{A} \beta$-induced cell death (Boyd-Kimball et al., 2005; Zampagni et al., 2012). However, whether these GSH precursors were efficient in increasing mGSH deserves further investigation.

In contrast to $\mathrm{AD}$, there has been very limited research on the role of mitochondrial cholesterol in other major neurodegenerative diseases, such as HD (Table 3), an autosomal-dominant neurodegenerative disorder caused by a CAG expansion in the Huntingtin (htt) gene. For instance, CAV1 knockout mice, which is characterized by increased mitochondrial cholesterol trafficking via MAM and subsequent mGSH depletion, exhibited increased brain damage caused by 3-nitropropionic acid (3NP) treatment (Bosch et al., 2011). 3-NP is a mitochondrial toxin used extensively as a model of $\mathrm{HD}$ and its toxicity is associated with oxidative stress. Injection of 3 -NP in the striatum of CAV1 null mice caused extensive cell degeneration as revealed by Fluoro-Jade stained serial sections compared to wild type mice. In addition, there is evidence that the multifunctional htt protein intimately interacts with a variety of lipid membranes, and is essential for the normal development of several perinuclear membrane organelles, including mitochondria and the ER (Gao et al., 2016). Moreover, brain-isolated mitochondrial membranes of HD models such as aged Hdh Q111/Q111 knock-in mice and BACHD transgenic rats as well as isolated from striatal STHdh Q111/Q111 cells, exhibited increased membrane fluidity (Eckmann et al., 2014). Intriguingly, however, both models of $\mathrm{HD}$ knock-in mice treated with olexosime, a cholesterol-like compound, showed decreasing membrane fluidity in brain-isolated mitochondrial membrane fractions and restored $\mathrm{HD}$-specific changes in mitochondrial membranes. Moreover, BACHD rats treated during 12-months with olexosime showed counteracted the mhtt-induced membrane fluidity increment of brain-isolated mitochondrial membranes. Although, these findings link altered mitochondrial membrane properties with HD and suggest that olexosime may be a potential therapeutic opportunity for HD, whether these effects are mediated by modulating mitochondrial cholesterol levels in OMM o IMM remains to be further investigated.

\section{MAM in Cholesterol Trafficking and Neurodegeneration}

As mentioned above MAM have emerged as specialized subdomains that play key roles in cholesterol and phospholipids metabolism, calcium signaling, apoptosis, ER stress, autophagy, and mitochondrial dynamics and integrity (Vance, 2014; van Vliet et al., 2014; Giorgi et al., 2015; Area-Gomez and Schon, 2016; van Vliet and Agostinis, 2017). Alterations in the signaling and/or components of MAMs are implicated in several neurodegenerative disorders including $\mathrm{AD}, \mathrm{PD}, \mathrm{HD}$, amyotrophic lateral sclerosis (ALS), frontotemporal dementia (FTD), and Charcot-Marie-Tooth disease (CMT; Giorgi et al., 2015; Krols et al., 2016; Area-Gomez and Schon, 2017). MAM is a specialized ER-subdomain that connects mitochondria and the ER, both physically and biochemically (Giorgi et al., 2015; Area-Gomez and Schon, 2016). This is quite relevant for neurons, since their morphology and function involve interorganelle communication via vesicular transport that depends on MAMs to exchange metabolites and signaling molecules. Importantly, it has been described that the ER portion of MAM is an intracellular lipid-raft domain given its detergentresistance (Area-Gomez et al., 2012). Moreover, PSEN1/2 and $\gamma$-secretase are located predominantly at the MAM (AreaGomez et al., 2012), in concordance with observations that localize $\mathrm{A} \beta$ within mitochondria in $\mathrm{AD}$-patients and transgenic AD-mice (Lustbader et al., 2004; Caspersen et al., 2005), and in neuroblastoma cells (Hayashi et al., 2012; Zheng et al., 2013). However, although there has been recent evidence against the support for the amyloidogenic APP processing in mitochondria from human neuroblastoma cell lines based on an iodixanol centrifugation gradient that separated mitochondria from lysosomes (Mamada et al., 2017), this approach disrupted the structure of MAM, which precluded functional analyses of APP processing in these domains. In contrast to these findings, MAMs isolated by a Percoll gradient centrifugation from cellular models overexpressing wild type APP or APP harboring familial $\mathrm{AD}$ mutations and from brains of double-transgenic $\mathrm{AD}$-mice 
model, revealed the presence of APP and its catabolites in conjunction with APP-processing active forms of both $\beta$ and $\gamma$-secretases (Del Prete et al., 2017). The impact APP processing in this subcellular localization was assessed by the overexpression of APP or BACE1 in human HEK293 cells (expressing low endogenous BACE1 expression) and N2a murine neuroblastoma cell line. APP overexpression (but not BACE1) reduced mitochondrial respiration and treatment with GSI to inhibit $\gamma$-secretase rescued this phenotype (Schaefer et al., 2016). Moreover, further analysis revealed that an impaired mitochondrial respiration is associated with both intracellular $\mathrm{A} \beta$ levels and an increased $\mathrm{APP} / \mathrm{A} \beta$-mitochondria apposition (Schaefer et al., 2016). In addition to $A \beta$-mediated mitochondrial toxicity, Del Prete and coworkers described that APP and A $\beta$ accumulated at MAM interact with key MAM's proteins that controls mitochondria and ER functions and contributes to an increase in ER-mitochondria contact sites (Del Prete et al., 2017). In fact, cells containing PSEN1mutant familial $\mathrm{AD}$ also showed MAM upregulation, which was mitigated by knocking down MFN2 (Area-Gomez et al., 2012), a MAM-resident protein involved in mitochondrial fusion and ER-mitochondrial connectivity. Moreover, PSEN1-mutant cells and $\mathrm{AD}$ fibroblasts exhibited a significant increase in the levels of both phosphatidylserine (synthesized at MAM) and phosphatidylethanolamine (converted at mitochondria; AreaGomez et al., 2012), indicating an increase in MAM activity, in concordance with the altered phospholipid profiles seen in $\mathrm{AD}$ (Mielke et al., 2010).

Interestingly, amyloidogenic processing of pathogenic APP at MAM seems to promote lipid droplets accumulation, and effect that is reversed upon inhibition of both $\beta$ - or $\gamma$-secretases (Del Prete et al., 2017). Indeed, this outcome of increased lipid droplets is consistent with the presence of higher activity of ACAT1, a MAM-resident protein, in both PSEN1-mutant cells and in cells from AD-patients compared with controls, correlating with increased lipid droplets (Area-Gomez et al., 2012). These findings are also consistent with the observation that ACAT1 activity is necessary for the production of $A \beta$ (Puglielli et al., 2004; Murphy et al., 2013; Zhu et al., 2015). For instance, both human fibroblasts and explanted mouse neurons treated with astrocyte-conditioned medium containing ApoE4 show a significant increase of MAM activity measured by both MAM-mediated phospholipid transport and ACAT1-mediated cholesteryl ester synthesis and lipid droplet formation (Tambini et al., 2016), suggesting a link between upregulation in MAM function and ApoE4 as a risk factor of AD (Figure 4).

Recently, among most key components of the cholesterol/steroid biosynthesis and transport pathways mapped from highly purified MAM fractions from mouse liver using in-depth mass spectrometry characterization; CAV1 was identified as a specific integral component of MAMs (Sala-Vila et al., 2016). Using a cytometry-based FRET assay through a rapamycin-inducible fluorescent artificial ER-mitochondria tethering reporters on embryonic fibroblast from wild-type and CAV1-deficient mice, Salas-Vila et al. observed a reduction in FRET signal in the CAV1-deficient cells indicating decreased MAM extension/stability associated with CAV1 deficiency that was accompanied by a significant increase in free cholesterol accumulation at MAM (Sala-Vila et al., 2016). Moreover, quantitative mass spectrometry showed alterations in steroid biosynthesis (depletion of HMGC synthase at MAM) and inorganic ion transport (MAM-enriched membrane cation transporters) in CAV1-deficient mice (Sala-Vila et al., 2016). Importantly, the absence of CAV1 has been related with aging and AD-like neuropathology, since similar to older WT mice, young CAV1-deficient mice exhibit reduction of synapses and degeneration (Head et al., 2010). Moreover, hippocampus from young CAV1-deficient mice show increased AD-like markers $(\mathrm{A} \beta, \mathrm{P}$-tau, astrogliosis, and neurodegeneration) compared with young WT mice (Head et al., 2010).

In addition to the regulation of lipid droplets, MAMs play a key role in transferring stress signals from the ER to mitochondria emerging as a central site involved in the upregulation of ER chaperones during the early adaptive phases of ER stress and in the regulation of steroidognesis (van Vliet and Agostinis, 2017). For instance, GRP78 has been localized in MAM in rat testes and MA-10 cells where facilitates StAR intermediate state folding that governs the folding state required for its delivery to OMM to promote steroidogenesis (Prasad et al., 2017). Indeed, siRNA-dependent GRP78 knockdown prevents StAR intermediate state emergence leading to the degradation of the unfolded StAR, which translates in the deletion of functional StAR and hence shutdown of mitochondrial steroidogenesis (Prasad et al., 2017). Thus, based on these findings, it is conceivable that GRP78 acts as an acute regulator of mitochondrial cholesterol loading and steroidogenesis at the MAM by regulating StAR folding. This outcome may be of relevance as GRP78 levels correlate with Braak staging of patients with $\mathrm{AD}$ and increased expression of GRP78 in AD models constitutes an early molecular event in the $A \beta$-induced ER stress leading to $\mathrm{mGSH}$ depletion through mitochondrial cholesterol loading, causing neurotoxicity and neuroinflammation (Barbero-Camps et al., 2014; Placido et al., 2014).

Together, all these observations not only support the concept that MAMs emerge as intracellular sites for amyloidogenic APP processing, but also that MAM agglutinates several other biochemical and morphological features typically observed at early stages of $\mathrm{AD}$ (Figure 2B), especially mitochondrial dysfunction as a common feature in many neurodegenerative diseases, defining the so called MAM hypothesis as a key event for AD pathogenesis (Area-Gomez and Schon, 2017).

\section{CONCLUDING REMARKS}

Cholesterol is a vital component of membrane bilayers that plays both structural and functional roles, regulating a wide range of signaling pathways and cell functions. Given these key biological functions, it seems evident that cholesterol homeostasis dysregulations have been associated with the onset of hereditary brain disorders and major neurodegenerative diseases. However, in the case of $\mathrm{AD}$, it is unclear whether increased or decreased brain cholesterol levels are involved in brain dysfunction and disease progression. 


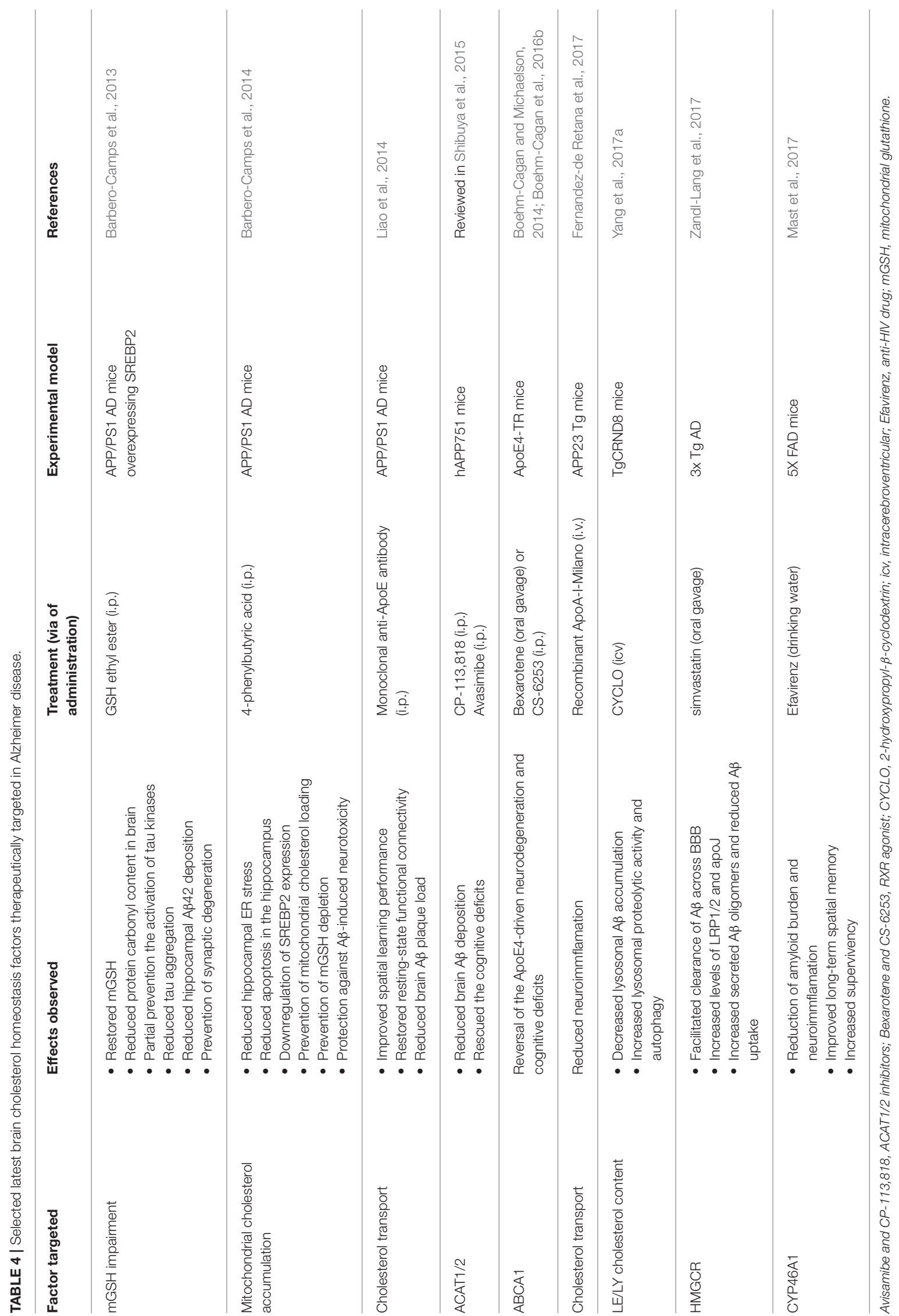


These findings suggest that the distribution may be more important than the alterations in total cholesterol levels for neurodegeneration. Indeed, cholesterol trafficking and accumulation in PM, endolysosomes and mitochondria via MAM may be more relevant to disease progression by regulating specific signaling pathways and intracellular compartment function. This premise has been recently well-illustrated with the case of mitochondrial cholesterol accumulation in NPC disease and AD disease. Although, the pathogenesis of both diseases is markedly different, both exhibit a common feature of increased cholesterol accumulation, which exceeds its metabolism within mitochondria. The accumulation of cholesterol in mitochondrial membranes, particularly in IMM, impairs the transport of GSH from cytosol to the mitochondrial matrix, rendering mitochondria more susceptible to $\mathrm{A} \beta$ induced ROS generation and neurotoxicity as well as in the modulation of kinase-dependent tau phosphorylation. The accumulation of mitochondrial cholesterol involved an ER stressmitochondrial cross-talk resulting in the increased expression of StARD1-mediated trafficking of cholesterol to IMM. A better understanding of the functional consequences and mechanisms of mitochondrial or endolysosomal enrichment in cholesterol may provide further insight in the pathogenesis of major neurodegenerative diseases and in the design of novel and improved therapies for treatment. In this regard, the Table 4 resume the latest approaches that therapeutically targeted several factors involved in the brain's cholesterol homeostasis regarding reduction/amelioration of neurodegeneration in animal models

\section{REFERENCES}

Allouche, M., Pertuiset, C., Robert, J. L., Martel, C., Veneziano, R., Henry, C., et al. (2012). ANT-VDAC1 interaction is direct and depends on ANT isoform conformation in vitro. Biochem. Biophys. Res. Commun. 429, 12-17. doi: 10.1016/j.bbrc.2012.10.108

Alpy, F., Stoeckel, M. E., Dierich, A., Escola, J. M., Wendling, C., Chenard, M. P., et al. (2001). The steroidogenic acute regulatory protein homolog MLN64, a late endosomal cholesterol-binding protein. J. Biol. Chem. 276, 4261-4269. doi: 10.1074/jbc.M006279200

Alpy, F., and Tomasetto, C. (2006). MLN64 and MENTHO, two mediators of endosomal cholesterol transport. Biochem. Soc. Trans. 34(Pt 3), 343-345. doi: 10.1042/BST0340343

Arbor, S. C., LaFontaine, M., and Cumbay, M. (2016). Amyloid-beta Alzheimer targets - protein processing, lipid rafts, and amyloid-beta pores. Yale J. Biol. Med. 89, 5-21.

Area-Gomez, E., Del Carmen Lara Castillo, M., Tambini, M. D., GuardiaLaguarta, C., de Groof, A. J., Madra, M., et al. (2012). Upregulated function of mitochondria-associated ER membranes in Alzheimer disease. EMBO J. 31, 4106-4123. doi: 10.1038/emboj.2012.202

Area-Gomez, E., and Schon, E. A. (2016). Mitochondria-associated ER membranes and Alzheimer disease. Curr. Opin. Genet. Dev. 38, 90-96. doi: 10.1016/j.gde.2016.04.006

Area-Gomez, E., and Schon, E. A. (2017). On the pathogenesis of Alzheimer's disease: the MAM hypothesis. FASEB J. 31, 864-867. doi: 10.1096/fj.201601309

Arrazola, M. S., Silva-Alvarez, C., and Inestrosa, N. C. (2015). How the Wnt signaling pathway protects from neurodegeneration: the mitochondrial scenario. Front. Cell. Neurosci. 9:166. doi: 10.3389/fncel.2015.00166

Balboa, E., Castro, J., Pinochet, M. J., Cancino, G. I., Matias, N., Jose Saez, P., et al. (2017). MLN64 induces mitochondrial dysfunction associated with increased mitochondrial cholesterol content. Redox Biol. 12, 274-284. doi: 10.1016/j.redox.2017.02.024 for AD. Among these approaches, improving mitochondrial antioxidant defense in a specific fashion may stand as a novel approach of relevance for neurodegeneration, such as $\mathrm{AD}$, and lysosomal disorders like NPC. In this regard, the boost of cytosolic GSH levels may be insufficient to guarantee mitochondrial GSH levels due to the restriction of increased mitochondrial cholesterol accumulation, which is bypassed by GSH permeable prodrugs that diffuse into mitochondria. The combination of this approach with other chemically modified scavengers targeted to mitochondria may be a promising approach for the treatment of neurodegenerative diseases.

\section{AUTHOR CONTRIBUTIONS}

FA, CG-R, and JF-C. discussed findings, analyzed literature, and wrote the manuscript.

\section{ACKNOWLEDGMENTS}

The work was supported by grants SAF-2014-57674-R, SAF2015-69944-R from Plan Nacional de I+D, Spain, Fundació Marató de TV3, La Mutua Madrileña, PI11/0325 (META) grant from the Instituto Salud Carlos III, and by the support of CIBEREHD; the center grant P50-AA-11999 Research Center for Liver and Pancretic Diseases funded by NIAAA/NIH; and support from AGAUR of the Generalitat de Catalunya 2014-SGR785.

Banati, R. B., Middleton, R. J., Chan, R., Hatty, C. R., Kam, W. W., Quin, C., et al. (2014). Positron emission tomography and functional characterization of a complete PBR/TSPO knockout. Nat. Commun. 5:5452. doi: $10.1038 /$ ncomms6452

Barbero-Camps, E., Fernandez, A., Baulies, A., Martinez, L., Fernandez-Checa, J. C., and Colell, A. (2014). Endoplasmic reticulum stress mediates amyloid beta neurotoxicity via mitochondrial cholesterol trafficking. Am. J. Pathol. 184, 2066-2081. doi: 10.1016/j.ajpath.2014.03.014

Barbero-Camps, E., Fernandez, A., Martinez, L., Fernandez-Checa, J. C., and Colell, A. (2013). APP/PS1 mice overexpressing SREBP-2 exhibit combined Abeta accumulation and tau pathology underlying Alzheimer's disease. Hum. Mol. Genet. 22, 3460-3476. doi: 10.1093/hmg/ddt201

Barbosa, A. D., and Siniossoglou, S. (2017). Function of lipid droplet-organelle interactions in lipid homeostasis. Biochim. Biophys. Acta 1864, 1459-1468. doi: 10.1016/j.bbamcr.2017.04.001

Barcelo-Coblijn, G., Golovko, M. Y., Weinhofer, I., Berger, J., and Murphy, E. J. (2007). Brain neutral lipids mass is increased in alpha-synuclein geneablated mice. J. Neurochem. 101, 132-141. doi: 10.1111/j.1471-4159.2006. 04348.x

Barrett, P. J., Song, Y., Van Horn, W. D., Hustedt, E. J., Schafer, J. M., Hadziselimovic, A., et al. (2012). The amyloid precursor protein has a flexible transmembrane domain and binds cholesterol. Science 336, 1168-1171. doi: 10.1126/science. 1219988

Bell, R. D., Winkler, E. A., Singh, I., Sagare, A. P., Deane, R., Wu, Z., et al. (2012). Apolipoprotein E controls cerebrovascular integrity via cyclophilin A. Nature 485, 512-516. doi: 10.1038/nature 11087

Boehm-Cagan, A., Bar, R., Harats, D., Shaish, A., Levkovitz, H., Bielicki, J. K., et al. (2016a). Differential effects of apoE4 and activation of ABCA1 on brain and plasma lipoproteins. PLoS ONE 11:e0166195. doi: 10.1371/journal.pone.0166195

Boehm-Cagan, A., Bar, R., Liraz, O., Bielicki, J. K., Johansson, J. O., and Michaelson, D. M. (2016b). ABCA1 agonist reverses the ApoE4-driven 
cognitive and brain pathologies. J. Alzheimers Dis. 54, 1219-1233. doi: 10.3233/JAD-160467

Boehm-Cagan, A., and Michaelson, D. M. (2014). Reversal of apoE4-driven brain pathology and behavioral deficits by bexarotene. J. Neurosci. 34, 7293-7301. doi: 10.1523/JNEUROSCI.5198-13.2014

Bosch, M., Mari, M., Gross, S. P., Fernandez-Checa, J. C., and Pol, A. (2011). Mitochondrial cholesterol: a connection between caveolin, metabolism, and disease. Traffic 12, 1483-1489. doi: 10.1111/j.1600-0854.2011.01259.x

Bosco, D. A., Fowler, D. M., Zhang, Q., Nieva, J., Powers, E. T., Wentworth, P. Jr., et al. (2006). Elevated levels of oxidized cholesterol metabolites in Lewy body disease brains accelerate alpha-synuclein fibrilization. Nat. Chem. Biol. 2, 249-253. doi: 10.1038/nchembio782

Boyd-Kimball, D., Sultana, R., Abdul, H. M., and Butterfield, D. A. (2005). $\gamma$-glutamylcysteine ethyl ester-induced up-regulation of glutathione protects neurons against $\mathrm{A} \beta(1-42)$-mediated oxidative stress and neurotoxicity: implications for Alzheimer's disease. J. Neurosci. Res. 79, 700-706. doi: 10.1002/jnr.20394

Caron, K. M., Soo, S. C., Wetsel, W. C., Stocco, D. M., Clark, B. J., and Parker, K. L. (1997). Targeted disruption of the mouse gene encoding steroidogenic acute regulatory protein provides insights into congenital lipoid adrenal hyperplasia. Proc. Natl. Acad. Sci. U.S.A. 94, 11540-11545. doi: 10.1073/pnas.94.21.11540

Caspersen, C., Wang, N., Yao, J., Sosunov, A., Chen, X., Lustbader, J. W., et al. (2005). Mitochondrial Abeta: a potential focal point for neuronal metabolic dysfunction in Alzheimer's disease. FASEB J. 19, 2040-2041. doi: 10.1096/fj.05-3735fje

Castagnet, P. I., Golovko, M. Y., Barcelo-Coblijn, G. C., Nussbaum, R. L., and Murphy, E. J. (2005). Fatty acid incorporation is decreased in astrocytes cultured from alpha-synuclein gene-ablated mice. J. Neurochem. 94, 839-849. doi: 10.1111/j.1471-4159.2005.03247.x

Cermak, S., Kosicek, M., Mladenovic-Djordjevic, A., Smiljanic, K., Kanazir, S., and Hecimovic, S. (2016). Loss of Cathepsin B and L leads to lysosomal dysfunction, NPC-Like cholesterol sequestration and accumulation of the key Alzheimer's proteins. PLoS ONE 11:e0167428. doi: 10.1371/journal.pone.0167428

Charman, M., Kennedy, B. E., Osborne, N., and Karten, B. (2010). MLN64 mediates egress of cholesterol from endosomes to mitochondria in the absence of functional Niemann-Pick Type C1 protein. J. Lipid Res. 51, 1023-1034. doi: 10.1194/jlr.M002345

Chen, D. W., Yang, J. F., Tang, Z., Dong, X. M., Feng, X. L., Yu, S., et al. (2008). Cholesteryl ester transfer protein polymorphism D442G associated with a potential decreased risk for Alzheimer's disease as a modifier for APOE epsilon 4 in Chinese. Brain Res. 1187, 52-57. doi: 10.1016/j.brainres.2007.10.054

Cheng, D., Kim, W. S., and Garner, B. (2008). Regulation of alpha-synuclein expression by liver X receptor ligands in vitro. Neuroreport 19, 1685-1689. doi: 10.1097/WNR.0b013e32831578b2

Cianciola, N. L., Greene, D. J., Morton, R. E., and Carlin, C. R. (2013). Adenovirus RIDalpha uncovers a novel pathway requiring ORP1L for lipid droplet formation independent of NPC1. Mol. Biol. Cell 24, 3309-3325. doi: $10.1091 / \mathrm{mbc} . \mathrm{E} 12-10-0760$

Cole, N. B., Murphy, D. D., Grider, T., Rueter, S., Brasaemle, D., and Nussbaum, R. L. (2002). Lipid droplet binding and oligomerization properties of the Parkinson's disease protein alpha-synuclein. J. Biol. Chem. 277, 6344-6352. doi: 10.1074/jbc.M108414200

Cole, S. L., Grudzien, A., Manhart, I. O., Kelly, B. L., Oakley, H., and Vassar, R. (2005). Statins cause intracellular accumulation of amyloid precursor protein, $\beta$-secretase-cleaved fragments, and amyloid $\beta$-peptide via an isoprenoid-dependent mechanism. J. Biol. Chem. 280, 18755-18770. doi: 10.1074/jbc.M413895200

Colin, J., Gregory-Pauron, L., Lanhers, M. C., Claudepierre, T., Corbier, C., Yen, F. T., et al. (2016). Membrane raft domains and remodeling in aging brain. Biochimie 130, 178-187. doi: 10.1016/j.biochi.2016.08.014

Das, U., Scott, D. A., Ganguly, A., Koo, E. H., Tang, Y., and Roy, S. (2013). Activity-induced convergence of APP and BACE-1 in acidic microdomains via an endocytosis-dependent pathway. Neuron 79, 447-460. doi: 10.1016/j.neuron.2013.05.035

Del Prete, D., Suski, J. M., Oules, B., Debayle, D., Gay, A. S., Lacas-Gervais, S., et al. (2017). Localization and processing of the amyloid-beta protein precursor in mitochondria-associated membranes. J. Alzheimers Dis. 55, 1549-1570. doi: $10.3233 /$ JAD-160953 de Oliveira, J., Hort, M. A., Moreira, E. L., Glaser, V., Ribeiro-do-Valle, R. M., Prediger, R. D., et al. (2011). Positive correlation between elevated plasma cholesterol levels and cognitive impairments in LDL receptor knockout mice: relevance of cortico-cerebral mitochondrial dysfunction and oxidative stress. Neuroscience 197, 99-106. doi: 10.1016/j.neuroscience.2011.09.009

Desai, R., Frazier, A. E., Durigon, R., Patel, H., Jones, A. W., Dalla Rosa, I., et al. (2017). ATAD3 gene cluster deletions cause cerebellar dysfunction associated with altered mitochondrial DNA and cholesterol metabolism. Brain 140, 1595-1610. doi: 10.1093/brain/awx094

Di Paolo, G., and Kim, T. W. (2011). Linking lipids to Alzheimer's disease: cholesterol and beyond. Nat. Rev. Neurosci. 12, 284-296. doi: 10.1038/nrn3012

Eckert, G. P., Cairns, N. J., Maras, A., Gattaz, W. F., and Muller, W. E. (2000). Cholesterol modulates the membrane-disordering effects of beta-amyloid peptides in the hippocampus: specific changes in Alzheimer's disease. Dement. Geriatr. Cogn. Disord. 11, 181-186. doi: 10.1159/000017234

Eckmann, J., Clemens, L. E., Eckert, S. H., Hagl, S., Yu-Taeger, L., Bordet, T., et al. (2014). Mitochondrial membrane fluidity is consistently increased in different models of Huntington disease: restorative effects of olesoxime. Mol. Neurobiol. 50, 107-118. doi: 10.1007/s12035-014-8663-3

Egawa, J., Pearn, M. L., Lemkuil, B. P., Patel, P. M., and Head, B. P. (2015). Membrane lipid rafts and neurobiology: age-related changes in membrane lipids and loss of neuronal function. J. Physiol. 594, 4565-4579. doi: 10.1113/JP270590

El Asmar, Z., Terrand, J., Jenty, M., Host, L., Mlih, M., Zerr, A., et al. (2016). Convergent signaling pathways controlled by LRP1 (receptor-related protein 1) cytoplasmic and extracellular domains limit cellular cholesterol accumulation. J. Biol. Chem. 291, 5116-5127. doi: 10.1074/jbc.M116.714485

El Gaamouch, F., Jing, P., Xia, J., and Cai, D. (2016). Alzheimer's disease risk genes and lipid regulators. J. Alzheimers Dis. 53, 15-29. doi: 10.3233/JAD-160169

Elustondo, P., Martin, L. A., and Karten, B. (2017). Mitochondrial cholesterol import. Biochim. Biophys. Acta 1862, 90-101. doi: 10.1016/j.bbalip.2016.08.012

Eriksson, I., Nath, S., Bornefall, P., Giraldo, A. M., and Ollinger, K. (2017). Impact of high cholesterol in a Parkinson's disease model: prevention of lysosomal leakage versus stimulation of alpha-synuclein aggregation. Eur. J. Cell Biol. 96, 99-109. doi: 10.1016/j.ejcb.2017.01.002

Fernandez, A., Llacuna, L., Fernandez-Checa, J. C., and Colell, A. (2009). Mitochondrial cholesterol loading exacerbates amyloid beta peptideinduced inflammation and neurotoxicity. J. Neurosci. 29, 6394-6405. doi: 10.1523/JNEUROSCI.4909-08.2009

Fernandez, A., Matias, N., Fucho, R., Ribas, V., Von Montfort, C., Nuno, N., et al. (2013). ASMase is required for chronic alcohol induced hepatic endoplasmic reticulum stress and mitochondrial cholesterol loading. J. Hepatol. 59, 805-813. doi: 10.1016/j.jhep.2013.05.023

Fernández-Checa, J. C. (2015). "Free Cholesterol - A Double-Edge Sword in Alzheimer Disease," in Alzheimer's Disease-Challenges for the Future, ed I. Zerr (Rijeka: InTechopen), 141-166. doi: 10.5772/59935

Fernandez-de Retana, S., Montanola, A., Marazuela, P., De La Cuesta, M., Batlle, A., Fatar, M., et al. (2017). Intravenous treatment with human recombinant ApoA-I Milano reduces beta amyloid cerebral deposition in the APP23transgenic mouse model of Alzheimer's disease. Neurobiol. Aging 60, 116-128. doi: 10.1016/j.neurobiolaging.2017.08.028

Galvagnion, C. (2017). The role of lipids interacting with alpha-synuclein in the pathogenesis of Parkinson's disease. J. Parkinsons Dis. 7, 433-450. doi: 10.3233/JPD-171103

Gamba, P., Testa, G., Gargiulo, S., Staurenghi, E., Poli, G., and Leonarduzzi, G. (2015). Oxidized cholesterol as the driving force behind the development of Alzheimer's disease. Front. Aging Neurosci. 7:119. doi: 10.3389/fnagi.2015.00119

Gao, X., Campbell, W. A., Chaibva, M., Jain, P., Leslie, A. E., Frey, S. L., et al. (2016). Cholesterol modifies huntingtin binding to, disruption of, and aggregation on lipid membranes. Biochemistry 55, 92-102. doi: 10.1021/acs.biochem. 5 b00900

Garcia-Ruiz, C., Mari, M., Colell, A., Morales, A., Caballero, F., Montero, J., et al. (2009). Mitochondrial cholesterol in health and disease. Histol. Histopathol. 24, 117-132. doi: 10.14670/HH-24.117

Gerhold, J. M., Cansiz-Arda, S., Lohmus, M., Engberg, O., Reyes, A., van Rennes, H., et al. (2015). Human mitochondrial DNA-protein complexes attach to a cholesterol-rich membrane structure. Sci. Rep. 5:15292. doi: 10.1038/srep15292 
Giorgi, C., Missiroli, S., Patergnani, S., Duszynski, J., Wieckowski, M. R., and Pinton, P. (2015). Mitochondria-associated membranes: composition, molecular mechanisms, and physiopathological implications. Antioxid. Redox Signal. 22, 995-1019. doi: 10.1089/ars.2014.6223

Goto, A., Charman, M., and Ridgway, N. D. (2016). Oxysterol-binding protein activation at endoplasmic reticulum-golgi contact sites reorganizes phosphatidylinositol 4-phosphate pools. J. Biol. Chem. 291, 1336-1347. doi: 10.1074/jbc.M115.682997

Gudala, K., Bansal, D., and Muthyala, H. (2013). Role of serum cholesterol in Parkinson's disease: a meta-analysis of evidence. J. Parkinsons Dis. 3, 363-370. doi: 10.3233/JPD-130196

Hayashi, H., Nakagami, H., Takeichi, M., Shimamura, M., Koibuchi, N., Oiki, E., et al. (2012). HIG1, a novel regulator of mitochondrial gammasecretase, maintains normal mitochondrial function. FASEB J. 26, 2306-2317. doi: 10.1096/fj.11-196063

Head, B. P., Peart, J. N., Panneerselvam, M., Yokoyama, T., Pearn, M. L., Niesman, I. R., et al. (2010). Loss of caveolin-1 accelerates neurodegeneration and aging. PLoS ONE 5:e15697. doi: 10.1371/journal.pone.0015697

Heverin, M., Bogdanovic, N., Lutjohann, D., Bayer, T., Pikuleva, I., Bretillon, L., et al. (2004). Changes in the levels of cerebral and extracerebral sterols in the brain of patients with Alzheimer's disease. J. Lipid Res. 45, 186-193. doi: 10.1194/jlr.M300320-JLR200

Hoozemans, J. J., van Haastert, E. S., Nijholt, D. A., Rozemuller, A. J., and Scheper, W. (2012). Activation of the unfolded protein response is an early event in Alzheimer's and Parkinson's disease. Neurodegener. Dis. 10, 212-215. doi: $10.1159 / 000334536$

Hu, G., Antikainen, R., Jousilahti, P., Kivipelto, M., and Tuomilehto, J. (2008). Total cholesterol and the risk of Parkinson disease. Neurology 70, 1972-1979. doi: 10.1212/01.wnl.0000312511.62699.a8

Huang, X., Auinger, P., Eberly, S., Oakes, D., Schwarzschild, M., Ascherio, A., et al. (2011). Serum cholesterol and the progression of Parkinson's disease: results from DATATOP. PLOS ONE 6:e22854. doi: 10.1371/journal.pone. 0022854

Ishii, T., Mitsui, T., Suzuki, S., Matsuzaki, Y., and Hasegawa, T. (2012). A genome-wide expression profile of adrenocortical cells in knockout mice lacking steroidogenic acute regulatory protein. Endocrinology 153, 2714-2723. doi: 10.1210/en.2011-1627

Kagedal, K., Kim, W. S., Appelqvist, H., Chan, S., Cheng, D., Agholme, L., et al. (2010). Increased expression of the lysosomal cholesterol transporter NPC1 in Alzheimer's disease. Biochim. Biophys. Acta 1801, 831-838. doi: 10.1016/j.bbalip.2010.05.005

Karten, B., Campenot, R. B., Vance, D. E., and Vance, J. E. (2006). Expression of ABCG1, but not ABCA1, correlates with cholesterol release by cerebellar astroglia. J. Biol. Chem. 281, 4049-4057. doi: 10.1074/jbc.M5089 15200

Kim, J., Castellano, J. M., Jiang, H., Basak, J. M., Parsadanian, M., Pham, V., et al. (2009). Overexpression of low-density lipoprotein receptor in the brain markedly inhibits amyloid deposition and increases extracellular A beta clearance. Neuron 64, 632-644. doi: 10.1016/j.neuron.2009.11.013

Kojro, E., Gimpl, G., Lammich, S., Marz, W., and Fahrenholz, F. (2001). Low cholesterol stimulates the nonamyloidogenic pathway by its effect on the alpha -secretase ADAM 10. Proc. Natl. Acad. Sci. U.S.A. 98, 5815-5820. doi: $10.1073 /$ pnas.081612998

Koldamova, R., Fitz, N. F., and Lefterov, I. (2014). ATP-binding cassette transporter A1: from metabolism to neurodegeneration. Neurobiol. Dis. 72(Pt A), 13-21. doi: 10.1016/j.nbd.2014.05.007

Kreilaus, F., Spiro, A. S., Hannan, A. J., Garner, B., and Jenner, A. M. (2015). Brain cholesterol synthesis and metabolism is progressively disturbed in the R6/1 mouse model of huntington's disease: a targeted GC-MS/MS sterol analysis. J. Huntingtons Dis. 4, 305-318. doi: 10.3233/JHD-150170

Krols, M., van Isterdael, G., Asselbergh, B., Kremer, A., Lippens, S., Timmerman, V., et al. (2016). Mitochondria-associated membranes as hubs for neurodegeneration. Acta Neuropathol. 131, 505-523. doi: 10.1007/s00401-015-1528-7

Kurup, R. K., and Kurup, P. A. (2003). Hypothalamic digoxin-mediated model for Parkinson's disease. Int. J. Neurosci. 113, 515-536. doi: $10.1080 / 00207450390162263$
Leoni, V., and Caccia, C. (2015). The impairment of cholesterol metabolism in Huntington disease. Biochim. Biophys. Acta 1851, 1095-1105. doi: 10.1016/j.bbalip.2014.12.018

Lev, S. (2010). Non-vesicular lipid transport by lipid-transfer proteins and beyond. Nat. Rev. Mol. Cell Biol. 11, 739-750. doi: 10.1038/nrm2971

Li, W., and Huang, E. (2016). An update on type 2 diabetes mellitus as a risk factor for Dementia. J. Alzheimers Dis. 53, 393-402. doi: 10.3233/JAD-160114

Liao, F., Hori, Y., Hudry, E., Bauer, A. Q., Jiang, H., Mahan, T. E., et al. (2014). AntiApoE antibody given after plaque onset decreases Abeta accumulation and improves brain function in a mouse model of Abeta amyloidosis. J. Neurosci. 34, 7281-7292. doi: 10.1523/JNEUROSCI.0646-14.2014

Lim, L., Jackson-Lewis, V., Wong, L. C., Shui, G. H., Goh, A. X., Kesavapany, S., et al. (2012). Lanosterol induces mitochondrial uncoupling and protects dopaminergic neurons from cell death in a model for Parkinson's disease. Cell Death Differ. 19, 416-427. doi: 10.1038/cdd.2011.105

Lin, M. T., and Beal, M. F. (2006). Alzheimer's APP mangles mitochondria. Nat. Med. 12, 1241-1243. doi: 10.1038/nm1106-1241

Lin, Y., Hou, X., Shen, W. J., Hanssen, R., Khor, V. K., Cortez, Y., et al. (2016). SNARE-mediated cholesterol movement to mitochondria supports steroidogenesis in rodent cells. Mol. Endocrinol. 30, 234-247. doi: $10.1210 /$ me.2015-1281

Liu, L., Zhang, K., Sandoval, H., Yamamoto, S., Jaiswal, M., Sanz, E., et al. (2015). Glial lipid droplets and ROS induced by mitochondrial defects promote neurodegeneration. Cell 160, 177-190. doi: 10.1016/j.cell.2014.12.019

Luo, J., Jiang, L., Yang, H., and Song, B. L. (2017). Routes and mechanisms of postendosomal cholesterol trafficking: a story that never ends. Traffic 18, 209-217. doi: $10.1111 /$ tra. 12471

Lustbader, J. W., Cirilli, M., Lin, C., Xu, H. W., Takuma, K., Wang, N., et al. (2004). ABAD directly links Abeta to mitochondrial toxicity in Alzheimer's disease. Science 304, 448-452. doi: 10.1126/science.1091230

Magalhaes, J., Gegg, M. E., Migdalska-Richards, A., Doherty, M. K., Whitfield, P. D., and Schapira, A. H. (2016). Autophagic lysosome reformation dysfunction in glucocerebrosidase deficient cells: relevance to Parkinson disease. Hum. Mol. Genet. 25, 3432-3445. doi: 10.1093/hmg/ddw185

Malnar, M., Hecimovic, S., Mattsson, N., and Zetterberg, H. (2014). Bidirectional links between Alzheimer's disease and Niemann-Pick type C disease. Neurobiol. Dis. 72(Pt A), 37-47. doi: 10.1016/j.nbd.2014.05.033

Malnar, M., Kosicek, M., Mitterreiter, S., Omerbasic, D., Lichtenthaler, S. F., Goate, A., et al. (2010). Niemann-Pick type C cells show cholesterol dependent decrease of APP expression at the cell surface and its increased processing through the beta-secretase pathway. Biochim. Biophys. Acta 1802, 682-691. doi: 10.1016/j.bbadis.2010.05.006

Mamada, N., Tanokashira, D., Ishii, K., Tamaoka, A., and Araki, W. (2017). Mitochondria are devoid of amyloid beta-protein (Abeta)producing secretases: evidence for unlikely occurrence within mitochondria of Abeta generation from amyloid precursor protein. Biochem. Biophys. Res. Commun. 486, 321-328. doi: 10.1016/j.bbrc.2017. 03.035

Mari, M., Caballero, F., Colell, A., Morales, A., Caballeria, J., Fernandez, A., et al. (2006). Mitochondrial free cholesterol loading sensitizes to TNF- and Fas-mediated steatohepatitis. Cell Metab. 4, 185-198. doi: 10.1016/j.cmet.2006.07.006

Martin, M. G., Pfrieger, F., and Dotti, C. G. (2014). Cholesterol in brain disease: sometimes determinant and frequently implicated. EMBO Rep. 15, 1036-1052. doi: 10.15252/embr.201439225

Martins, I. J., Berger, T., Sharman, M. J., Verdile, G., Fuller, S. J., and Martins, R. N. (2009). Cholesterol metabolism and transport in the pathogenesis of Alzheimer's disease. J. Neurochem. 111, 1275-1308.

Mason, R. P., Shoemaker, W. J., Shajenko, L., Chambers, T. E., and Herbette, L. G. (1992). Evidence for changes in the Alzheimer's disease brain cortical membrane structure mediated by cholesterol. Neurobiol. Aging 13, 413-419.

Mast, N., Saadane, A., Valencia-Olvera, A., Constans, J., Maxfield, E., Arakawa, H., et al. (2017). Cholesterol-metabolizing enzyme cytochrome P450 46A1 as a pharmacologic target for Alzheimer's disease. Neuropharmacology 123, 465-476. doi: 10.1016/j.neuropharm.2017.06.026

Maulik, M., Peake, K., Chung, J., Wang, Y., Vance, J. E., and Kar, S. (2015). APP overexpression in the absence of NPC1 exacerbates metabolism 
of amyloidogenic proteins of Alzheimer's disease. Hum. Mol. Genet. 24, 7132-7150. doi: 10.1093/hmg/ddv413

Mehrotra, A., Sood, A., and Sandhir, R. (2015). Mitochondrial modulators improve lipid composition and attenuate memory deficits in experimental model of Huntington's disease. Mol. Cell. Biochem. 410, 281-292. doi: 10.1007/s11010-015-2561-5

Mesmin, B., Antonny, B., and Drin, G. (2013). Insights into the mechanisms of sterol transport between organelles. Cell. Mol. Life Sci. 70, 3405-3421. doi: 10.1007/s00018-012-1247-3

Mielke, M. M., Haughey, N. J., Ratnam Bandaru, V. V., Schech, S., Carrick, R., Carlson, M. C., et al. (2010). Plasma ceramides are altered in mild cognitive impairment and predict cognitive decline and hippocampal volume loss. Alzheimers Dement. 6, 378-385. doi: 10.1016/j.jalz.2010.03.014

Morohaku, K., Pelton, S. H., Daugherty, D. J., Butler, W. R., Deng, W., and Selvaraj, V. (2014). Translocator protein/peripheral benzodiazepine receptor is not required for steroid hormone biosynthesis. Endocrinology 155, 89-97. doi: 10.1210/en.2013-1556

Moutinho, M., Nunes, M. J., and Rodrigues, E. (2016). Cholesterol 24-hydroxylase: brain cholesterol metabolism and beyond. Biochim. Biophys. Acta 1861(12 Pt A), 1911-1920. doi: 10.1016/j.bbalip.2016.09.011

Murphy, S. R., Chang, C. C., Dogbevia, G., Bryleva, E. Y., Bowen, Z., Hasan, M. T., et al. (2013). Acat1 knockdown gene therapy decreases amyloidbeta in a mouse model of Alzheimer's disease. Mol. Ther. 21, 1497-1506. doi: $10.1038 / \mathrm{mt} .2013 .118$

Musanti, R., Parati, E., Lamperti, E., and Ghiselli, G. (1993). Decreased cholesterol biosynthesis in fibroblasts from patients with Parkinson disease. Biochem. Med. Metab. Biol. 49, 133-142. doi: 10.1006/bmmb.1993.1016

Ng, E. L., and Tang, B. L. (2008). Rab GTPases and their roles in brain neurons and glia. Brain Res. Rev. 58, 236-246. doi: 10.1016/j.brainresrev.2008.04.006

Notkola, I. L., Sulkava, R., Pekkanen, J., Erkinjuntti, T., Ehnholm, C., Kivinen, P., et al. (1998). Serum total cholesterol, apolipoprotein E epsilon 4 allele, and Alzheimer's disease. Neuroepidemiology 17, 14-20.

Olkkonen, V. M., and Li, S. (2013). Oxysterol-binding proteins: sterol and phosphoinositide sensors coordinating transport, signaling and metabolism. Prog. Lipid Res. 52, 529-538. doi: 10.1016/j.plipres.2013.06.004

Pani, A., Dessì, S., Diaz, G., La Colla, P., Abete, C., Mulas, C., et al. (2009). Altered cholesterol ester cycle in skin fibroblasts from patients with Alzheimer's disease. J. Alzheimers Dis. 18, 829-841. doi: 10.3233/JAD-2009-1193

Paul, R., Choudhury, A., and Borah, A. (2015). Cholesterol - A putative endogenous contributor towards Parkinson's disease. Neurochem. Int. 90, 125-133. doi: 10.1016/j.neuint.2015.07.025

Paul, R., Choudhury, A., Kumar, S., Giri, A., Sandhir, R., and Borah, A. (2017). Cholesterol contributes to dopamine-neuronal loss in MPTP mouse model of Parkinson's disease: involvement of mitochondrial dysfunctions and oxidative stress. PLoS ONE 12:e0171285. doi: 10.1371/journal.pone.0171285

Persson, T., Lattanzio, F., Calvo-Garrido, J., Rimondini, R., Rubio-Rodrigo, M., Sundstrom, E., et al. (2017). Apolipoprotein E4 elicits lysosomal cathepsin D release, decreased thioredoxin-1 levels, and apoptosis. J. Alzheimers. Dis. 56, 601-617. doi: 10.3233/JAD-150738

Petanceska, S. S., DeRosa, S., Sharma, A., Diaz, N., Duff, K., Tint, S. G., et al. (2003). Changes in apolipoprotein E expression in response to dietary and pharmacological modulation of cholesterol. J. Mol. Neurosci. 20, 395-406. doi: $10.1385 /$ JMN:20:3:395

Placido, A. I., Pereira, C. M., Duarte, A. I., Candeias, E., Correia, S. C., Santos, R. X., et al. (2014). The role of endoplasmic reticulum in amyloid precursor protein processing and trafficking: implications for Alzheimer's disease. Biochim. Biophys. Acta 1842, 1444-1453. doi: 10.1016/j.bbadis.2014.05.003

Prasad, M., Kaur, J., Pawlak, K. J., Bose, M., Whittal, R. M., and Bose, H. S. (2015). Mitochondria-associated endoplasmic reticulum membrane (MAM) regulates steroidogenic activity via steroidogenic acute regulatory protein (StAR)-voltage-dependent anion channel 2 (VDAC2) interaction. J. Biol. Chem. 290, 2604-2616. doi: 10.1074/jbc.M114.605808

Prasad, M., Pawlak, K. J., Burak, W. E., Perry, E. E., Marshall, B., Whittal, R. M., et al. (2017). Mitochondrial metabolic regulation by GRP78. Sci. Adv. 3:e1602038. doi: 10.1126/sciadv.1602038

Puglielli, L., Ellis, B. C., Ingano, L. A., and Kovacs, D. M. (2004). Role of acylcoenzyme a: cholesterol acyltransferase activity in the processing of the amyloid precursor protein. J. Mol. Neurosci. 24, 93-96. doi: 10.1385/JMN:24:1:093
Ramanathan, A., Nelson, A. R., Sagare, A. P., and Zlokovic, B. V. (2015). Impaired vascular-mediated clearance of brain amyloid beta in Alzheimer's disease: the role, regulation and restoration of LRP1. Front. Aging Neurosci. 7:136. doi: 10.3389/fnagi.2015.00136

Refolo, L. M., Malester, B., LaFrancois, J., Bryant-Thomas, T., Wang, R., Tint, G. S., et al. (2000). Hypercholesterolemia accelerates the Alzheimer's amyloid pathology in a transgenic mouse model. Neurobiol. Dis. 7, 321-331. doi: 10.1006/nbdi.2000.0304

Ribas, V., Garcia-Ruiz, C., and Fernandez-Checa, J. C. (2014). Glutathione and mitochondria. Front. Pharmacol. 5:151. doi: 10.3389/fphar.2014.00151

Saeedi Saravi, S. S., Saeedi Saravi, S. S., Khoshbin, K., and Dehpour, A. R. (2017). Current insights into pathogenesis of Parkinson's disease: approach to mevalonate pathway and protective role of statins. Biomed. Pharmacother. 90, 724-730. doi: 10.1016/j.biopha.2017.04.038

Sala-Vila, A., Navarro-Lerida, I., Sanchez-Alvarez, M., Bosch, M., Calvo, C., Lopez, J. A., et al. (2016). Interplay between hepatic mitochondria-associated membranes, lipid metabolism and caveolin-1 in mice. Sci. Rep. 6:27351. doi: 10.1038/srep27351

Salvioli, R., Tatti, M., Scarpa, S., Moavero, S. M., Ciaffoni, F., Felicetti, F., et al. (2005). The N370S (Asn370->Ser) mutation affects the capacity of glucosylceramidase to interact with anionic phospholipidcontaining membranes and saposin C. Biochem. J. 390(Pt 1), 95-103. doi: 10.1042/BJ20050325

Schaaf, C. P., Koster, J., Katsonis, P., Kratz, L., Shchelochkov, O. A., Scaglia, F., et al. (2011). Desmosterolosis-phenotypic and molecular characterization of a third case and review of the literature. Am. J. Med. Genet. A 155A, 1597-1604. doi: 10.1002/ajmg.a.34040

Schaefer, P. M., von Einem, B., Walther, P., Calzia, E., and von Arnim, C. A. (2016). Metabolic characterization of intact cells reveals intracellular amyloid beta but not its precursor protein to reduce mitochondrial respiration. PLoS ONE 11:e0168157. doi: 10.1371/journal.pone.0168157

Schon, E. A., and Area-Gomez, E. (2013). Mitochondria-associated ER membranes in Alzheimer disease. Mol. Cell. Neurosci. 55, 26-36. doi: 10.1016/j.mcn.2012.07.011

Schultz, M. L., Krus, K. L., and Lieberman, A. P. (2016). Lysosome and endoplasmic reticulum quality control pathways in Niemann-Pick type C disease. Brain Res. 1649(Pt B), 181-188. doi: 10.1016/j.brainres.2016.03.035

Schweinzer, C., Kober, A., Lang, I., Etschmaier, K., Scholler, M., Kresse, A., et al. (2011). Processing of endogenous AbetaPP in blood-brain barrier endothelial cells is modulated by liver-X receptor agonists and altered cellular cholesterol homeostasis. J. Alzheimers. Dis. 27, 341-360. doi: 10.3233/JAD-2011-110854

Segatto, M., Leboffe, L., Trapani, L., and Pallottini, V. (2014). Cholesterol homeostasis failure in the brain: implications for synaptic dysfunction and cognitive decline. Curr. Med. Chem. 21, 2788-2802. doi: 10.2174/0929867321666140303142902

Selvaraj, V., and Tu, L. N. (2016). Current status and future perspectives: TSPO in steroid neuroendocrinology. J. Endocrinol. 231, R1-R30. doi: 10.1530/JOE-16-0241

Shankaran, M., Di Paolo, E., Leoni, V., Caccia, C., Ferrari Bardile, C., Mohammed, H., et al. (2017). Early and brain region-specific decrease of de novo cholesterol biosynthesis in Huntington's disease: a cross-validation study in Q175 knock-in mice. Neurobiol. Dis. 98, 66-76. doi: 10.1016/j.nbd.2016.11.013

Shibuya, Y., Chang, C. C., and Chang, T. Y. (2015). ACAT1/SOAT1 as a therapeutic target for Alzheimer's disease. Future Med. Chem. 7, 2451-2467. doi: 10.4155/fmc.15.161

Shie, F. S., Jin, L. W., Cook, D. G., Leverenz, J. B., and LeBoeuf, R. C. (2002). Diet-induced hypercholesterolemia enhances brain A beta accumulation in transgenic mice. Neuroreport 13, 455-459.

Sims-Robinson, C., Bakeman, A., Rosko, A., Glasser, R., and Feldman, E. L. (2016). The role of oxidized cholesterol in diabetes-induced lysosomal dysfunction in the brain. Mol. Neurobiol. 53, 2287-2296. doi: 10.1007/s12035-015-9207-1

Soffientini, U., and Graham, A. (2016). Intracellular cholesterol transport proteins: roles in health and disease. Clin. Sci. 130, 1843-1859. doi: 10.1042/CS20 160339

Sparks, D. L., Scheff, S. W., Hunsaker, J. C. III, Liu, H., Landers, T., and Gross, D. R. (1994). Induction of Alzheimer-like beta-amyloid immunoreactivity in the brains of rabbits with dietary cholesterol. Exp. Neurol. 126, 88-94. doi: 10.1006/exnr.1994.1044 
Stocco, D. M., Zhao, A. H., Tu, L. N., Morohaku, K., and Selvaraj, V. (2017). A brief history of the search for the protein(s) involved in the acute regulation of steroidogenesis. Mol. Cell. Endocrinol. 441, 7-16. doi: 10.1016/j.mce.2016.07.036

Tambini, M. D., Pera, M., Kanter, E., Yang, H., Guardia-Laguarta, C., Holtzman, D., et al. (2016). ApoE4 upregulates the activity of mitochondria-associated ER membranes. EMBO Rep. 17, 27-36. doi: 10.15252/embr.201540614

Tan, L. C., Methawasin, K., Tan, E. K., Tan, J. H., Au, W. L., Yuan, J. M., et al. (2016). Dietary cholesterol, fats and risk of Parkinson's disease in the Singapore chinese health study. J. Neurol. Neurosurg. Psychiatr. 87, 86-92. doi: 10.1136/jnnp-2014-310065

Theendakara, V., Peters-Libeu, C. A., Spilman, P., Poksay, K. S., Bredesen, D. E., and Rao, R. V. (2016). Direct transcriptional effects of apolipoprotein E. J. Neurosci. 36, 685-700. doi: 10.1523/JNEUROSCI.3562-15.2016

Thirumangalakudi, L., Prakasam, A., Zhang, R., Bimonte-Nelson, H., Sambamurti, K., Kindy, M. S., et al. (2008). High cholesterol-induced neuroinflammation and amyloid precursor protein processing correlate with loss of working memory in mice. J. Neurochem. 106, 475-485. doi: 10.1111/j.1471-4159.2008.05415.x

Thorsell, A. G., Lee, W. H., Persson, C., Siponen, M. I., Nilsson, M., Busam, R. D., et al. (2011). Comparative structural analysis of lipid binding START domains, PLoS ONE 6:e19521. doi: 10.1371/journal.pone.0019521

Torres, S., Matias, N., Baulies, A., Nunez, S., Alarcon-Vila, C., Martinez, L., et al. (2017). Mitochondrial GSH replenishment as a potential therapeutic approach for Niemann Pick type C disease. Redox Biol. 11, 60-72. doi: 10.1016/j.redox.2016.11.010

Tu, L. N., Morohaku, K., Manna, P. R., Pelton, S. H., Butler, W. R., Stocco, D. M., et al. (2014). Peripheral benzodiazepine receptor/translocator protein global knock-out mice are viable with no effects on steroid hormone biosynthesis. J. Biol. Chem. 289, 27444-27454. doi: 10.1074/jbc.M114.578286

Tu, L. N., Zhao, A. H., Stocco, D. M., and Selvaraj, V. (2015). PK11195 effect on steroidogenesis is not mediated through the translocator protein (TSPO). Endocrinology 156, 1033-1039. doi: 10.1210/en.2014-1707

Ujiie, M., Dickstein, D. L., Carlow, D. A., and Jefferies, W. A. (2003). Blood-brain barrier permeability precedes senile plaque formation in an Alzheimer disease model. Microcirculation 10, 463-470. doi: 10.1038/sj.mn.7800212

Vance, J. E. (2012). Dysregulation of cholesterol balance in the brain: contribution to neurodegenerative diseases. Dis. Model. Mech. 5, 746-755. doi: $10.1242 / \mathrm{dmm} .010124$

Vance, J. E. (2014). MAM (mitochondria-associated membranes) in mammalian cells: lipids and beyond. Biochim. Biophys. Acta 1841, 595-609. doi: 10.1016/j.bbalip.2013.11.014

van der Kant, R., Zondervan, I., Janssen, L., and Neefjes, J. (2013). Cholesterolbinding molecules MLN64 and ORP1L mark distinct late endosomes with transporters ABCA3 and NPC1. J. Lipid Res. 54, 2153-2165. doi: 10.1194/jlr.M037325

van der Wulp, M. Y., Verkade, H. J., and Groen, A. K. (2013). Regulation of cholesterol homeostasis. Mol. Cell. Endocrinol. 368, 1-16. doi: 10.1016/j.mce.2012.06.007

van Vliet, A. R., and Agostinis, P. (2017). "Mitochondria-associated membranes and ER stress," in Current Topics in Microbiology and Immunology, eds R. W. Compans, T. Honjo, B. Malissen, K. Aktories, J. E. Galan, R. Ahmed, K. Palme, A. Casadevall, and A. Garcia-Sastre (Berlin; Heidelberg: Springer), doi: 10.1007/82_2017_2

van Vliet, A. R., Verfaillie, T., and Agostinis, P. (2014). New functions of mitochondria associated membranes in cellular signaling. Biochim. Biophys. Acta 1843, 2253-2262. doi: 10.1016/j.bbamcr.2014.03.009

Webber, K. M., Stocco, D. M., Casadesus, G., Bowen, R. L., Atwood, C. S., Previll, L. A., et al. (2006). Steroidogenic acute regulatory protein (StAR): evidence of gonadotropin-induced steroidogenesis in Alzheimer disease. Mol. Neurodegener. 1:14. doi: 10.1186/1750-1326-1-14

Wolozin, B., Kellman, W., Ruosseau, P., Celesia, G. G., and Siegel, G. (2000). Decreased prevalence of Alzheimer disease associated with 3-hydroxy-3methyglutaryl coenzyme A reductase inhibitors. Arch. Neurol. 57, 1439-1443.
Xiang, Z., Valenza, M., Cui, L., Leoni, V., Jeong, H. K., Brilli, E., et al. (2011). Peroxisome-proliferator-activated receptor gamma coactivator 1 alpha contributes to dysmyelination in experimental models of Huntington's disease. J. Neurosci. 31, 9544-9553. doi: 10.1523/JNEUROSCI.1291-11.2011

Yamanaka, K., Urano, Y., Takabe, W., Saito, Y., and Noguchi, N. (2014). Induction of apoptosis and necroptosis by 24(S)-hydroxycholesterol is dependent on activity of acyl-CoA:cholesterol acyltransferase 1. Cell Death Dis. 5:e990. doi: 10.1038/cddis.2013.524

Yang, D. S., Stavrides, P., Kumar, A., Jiang, Y., Mohan, P. S., Ohno, M., et al. (2017a). Cyclodextrin has conflicting actions on autophagy flux in vivo in brains of normal and Alzheimer model mice. Hum. Mol. Genet. 26, 843-859. doi: $10.1093 / \mathrm{hmg} / \mathrm{ddx} 001$

Yang, H., Wang, Y., and Kar, S. (2017b). Effects of cholesterol transport inhibitor U18666A on APP metabolism in rat primary astrocytes. Glia 65, 1728-1743. doi: 10.1002/glia.23191

Yang, X., Sun, G. Y., Eckert, G. P., and Lee, J. C. (2014). Cellular membrane fluidity in amyloid precursor protein processing. Mol. Neurobiol. 50, 119-129. doi: 10.1007/s12035-014-8652-6

Yin, G., Lopes da Fonseca, T., Eisbach, S. E., Anduaga, A. M., Breda, C., Orcellet, M. L., et al. (2014). alpha-Synuclein interacts with the switch region of Rab8a in a Ser129 phosphorylation-dependent manner. Neurobiol. Dis. 70, 149-161. doi: 10.1016/j.nbd.2014.06.018

Yu, W., Gong, J. S., Ko, M., Garver, W. S., Yanagisawa, K., and Michikawa, M. (2005). Altered cholesterol metabolism in Niemann-Pick type C1 mouse brains affects mitochondrial function. J. Biol. Chem. 280, 11731-11739. doi: $10.1074 /$ jbc.M412898200

Zampagni, M., Wright, D., Cascella, R., D’Adamio, G., Casamenti, F., Evangelisti, E., et al. (2012). Novel S-acyl glutathione derivatives prevent amyloid oxidative stress and cholinergic dysfunction in Alzheimer disease models. Free Radic Biol. Med. 52, 1362-1371. doi: 10.1016/j.freeradbiomed.2012.01.012

Zandl-Lang, M., Fanaee-Danesh, E., Sun, Y., Cancar, I., Gali, C. C., Kober, A., et al. (2017). Regulatory effects of simvastatin and apoJ on APP processing and amyloid-beta clearance in blood-brain barrier endothelial cells. Biochim. Biophys. Acta 1863, 40-60. doi: 10.1016/j.bbalip.2017.09.008

Zhang, J., and Liu, Q. (2015). Cholesterol metabolism and homeostasis in the brain. Protein Cell 6, 254-264. doi: 10.1007/s13238-014-0131-3

Zhang, S., Glukhova, S. A., Caldwell, K. A., and Caldwell, G. A. (2017). NCEH-1 modulates cholesterol metabolism and protects against alpha-synuclein toxicity in a C. elegans model of Parkinson's disease. Hum. Mol. Genet. 26, 3823-3836. doi: $10.1093 / \mathrm{hmg} / \mathrm{ddx} 269$

Zheng, L., Calvo-Garrido, J., Hallbeck, M., Hultenby, K., Marcusson, J., Cedazo-Minguez, A., et al. (2013). Intracellular localization of amyloid-beta peptide in SH-SY5Y neuroblastoma cells. J. Alzheimers. Dis. 37, 713-733. doi: 10.3233/JAD-122455

Zhou, W., Scott, S. A., Shelton, S. B., and Crutcher, K. A. (2006). Cathepsin Dmediated proteolysis of apolipoprotein E: possible role in Alzheimer's disease. Neuroscience 143, 689-701. doi: 10.1016/j.neuroscience.2006.08.019

Zhu, M., Zhao, X., Chen, J., Xu, J., Hu, G., Guo, D., et al. (2015). ACAT1 regulates the dynamics of free cholesterols in plasma membrane which leads to the APP-alpha-processing alteration. Acta Biochim. Biophys. Sin. 47, 951-959. doi: 10.1093/abbs/gmv101

Zlokovic, B. V. (2010). Neurodegeneration and the neurovascular unit. Nat. Med. 16, 1370-1371. doi: 10.1038/nm1210-1370

Conflict of Interest Statement: The authors declare that the research was conducted in the absence of any commercial or financial relationships that could be construed as a potential conflict of interest.

Copyright (C) 2017 Arenas, Garcia-Ruiz and Fernandez-Checa. This is an open-access article distributed under the terms of the Creative Commons Attribution License (CC $B Y)$. The use, distribution or reproduction in other forums is permitted, provided the original author(s) or licensor are credited and that the original publication in this journal is cited, in accordance with accepted academic practice. No use, distribution or reproduction is permitted which does not comply with these terms. 